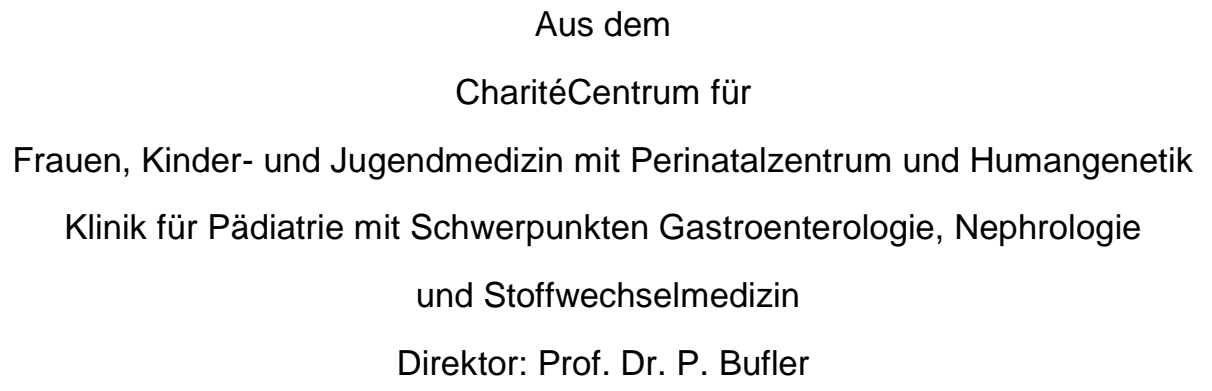

Habilitationsschrift

\title{
Intensivierte Hämodialyseverfahren
}

\section{bei Kindern und Jugendlichen}

\author{
zur Erlangung der Lehrbefähigung \\ für das Fach Kinder- und Jugendmedizin \\ vorgelegt dem Fakultätsrat der Medizinischen Fakultät \\ Charité-Universitätsmedizin Berlin \\ von \\ Dr. Julia Thumfart
}

\author{
Eingereicht: $\quad 01 / 2019$ \\ Dekan: $\quad$ Prof. Dr. med. Axel R. Pries \\ 1. Gutachter: Prof. Dr. Lutz Weber \\ 2. Gutachter: Prof. Dr. Christoph Aufricht
}


"Hemodialysis treatment time and $K t / V$ can both be considered to be primary measures of hemodialysis adequacy, because when either goes to zero, mortality is certain in patients without residual kidney function." 


\section{Inhaltsverzeichnis}

\section{Einleitung}

1.1. Terminale Niereninsuffizienz bei Kindern und Jugendlichen .........................................

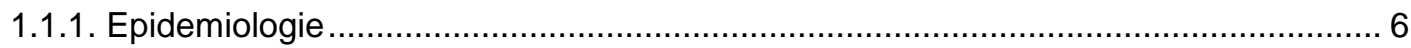

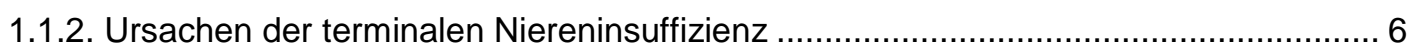

1.2. Indikation zum Beginn der Nierenersatztherapie bei Kindern und Jugendlichen ...............9

1.3. Wahl des Dialyseverfahrens bei Kindern und Jugendlichen ....................................... 12

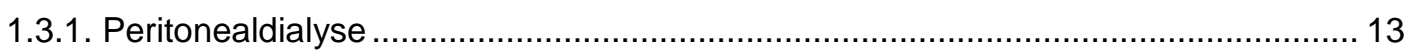

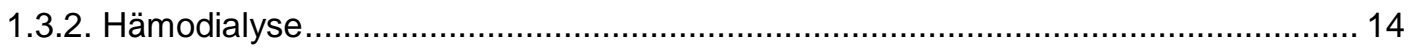

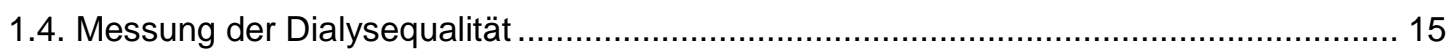

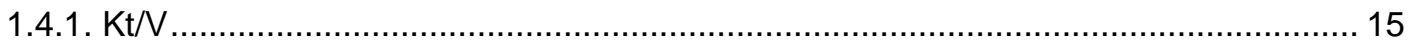

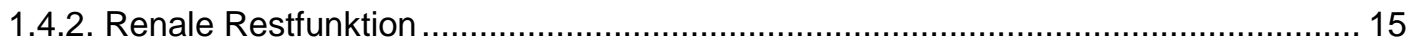

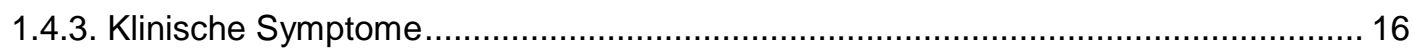

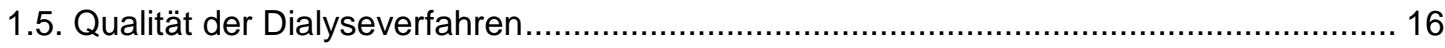

1.6. Ansätze zur Verbesserung der Dialysequalität ......................................................... 17

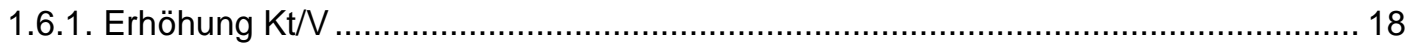

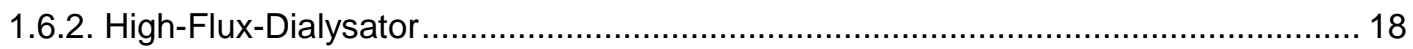

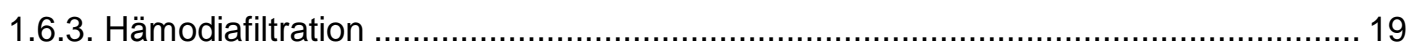

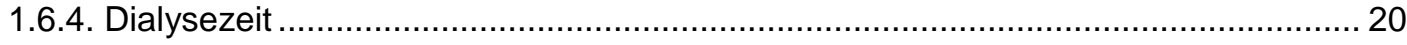

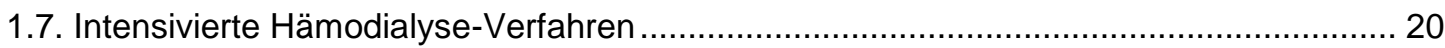

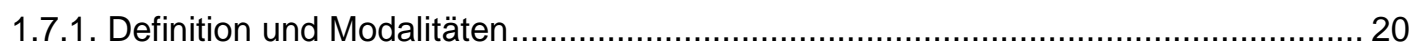

1.7.2. Rationale intensivierter Hämodialyseverfahren .................................................. 21

1.7.3. Intensivierte Hämodialyseverfahren bei Erwachsenen ........................................... 22

1.7.4. Intensivierte Dialyseverfahren bei Kindern und Jugendlichen ............................... 25

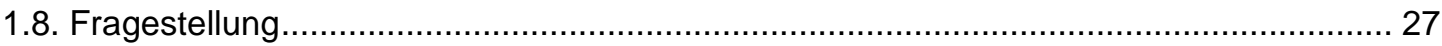

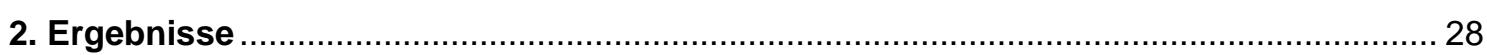

2.1. Aufbau eines Zentrums-basierten, nächtlich intermittierenden Hämodialyseprogramms für Kinder und Jugendliche („A hospital-based intermittent nocturnal hemodialysis program for children and adolescents") 28 
2.2. Hämodiafiltration in einem Zentrum-basierten nächtlich intermittierenden Dialyseprogramm für Kinder und Jugendliche („Hemodiafiltration in a pediatric nocturnal dialysis program").

2.3. Vergleich der nächtlichen Peritonealdialyse mit nächtlich intermittierender Hämodialyse bei Kinder und Jugendlichen (,Is peritoneal dialysis still an equal option? Results of the Berlin pediatric nocturnal dialysis program")

2.4. Implementierung und Hindernisse von intensivierten Hämodialyseverfahren bei Kindern und Jugendlichen weltweit („Barriers for implementation of intensified hemodialysis: survey results from the International Pediatric Dialysis Network")..... 50

2.5. Implementierung und Hindernisse von intensivierten Hämodialyseverfahren in Deutschland („Attitudes of nephrologists towards intensified hemodialysis“) ....... 59

2.6. Implementierung von assistierten Dialyseverfahren in Deutschland („Attitudes of

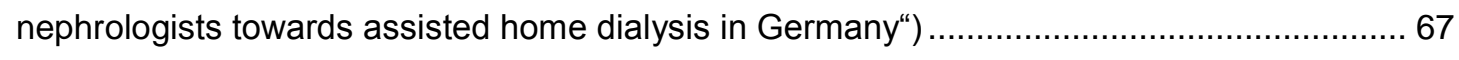

3. Diskussion 74

3.1. Kardiovaskuläre Surrogat-Parameter bei intensivierten HD-Verfahren 74

3.2. Lebensqualität bei intensivierten HD-Verfahren ....................................................... 76

3.3. Vor- und Nachteile der einzelnen intensivierten HD-Verfahren .................................... 78

3.4. Einsatz von intensivierten HD-Verfahren in der gegenwärtigen Praxis .......................... 80

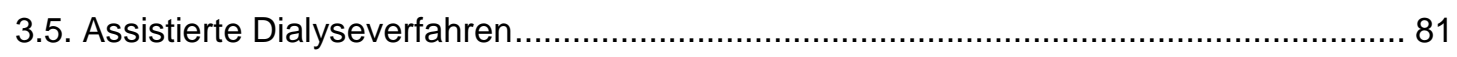

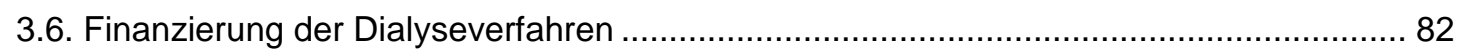

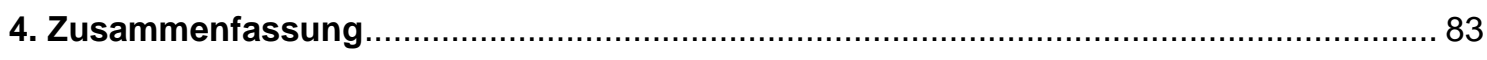

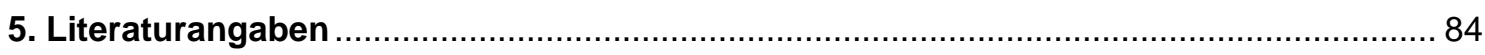

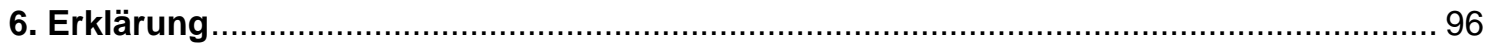

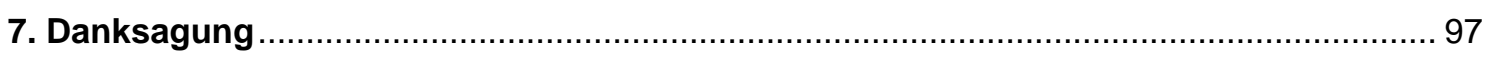




$\begin{array}{ll}\text { Abkürzungen } & \\ \text { ACTIVE } & \text { A clinical trial of intensive dialysis } \\ \text { ADEMEX } & \text { Adequacy of peritoneal dialysis in Mexico } \\ \text { ADPKD } & \text { Autosomal dominant polycystic kidney disease } \\ \text { ARPKD } & \text { Autosomal recessive polycystic kidney disease } \\ \text { AV-Fistel } & \text { Arteriovenöse Fistel } \\ \text { EBM } & \text { Einheitlicher Bewertungsmaßstab } \\ \text { CAKUT } & \text { Congenital anomalies of kidney and urinary tract } \\ \text { CAPD } & \text { Continuierliche ambulante Peritonealdialyse } \\ \text { CONTRAST } & \text { Convective transport study } \\ \text { CNI } & \text { Chronische Niereninsuffizienz } \\ \text { DGfN } & \text { Deutsche Gesellschaft für Nephrologie } \\ \text { DOPPS } & \text { Dialysis outcome practice pattern study } \\ \text { ESHOL } & \text { Estudio de supervivencia de hemodiafiltración On-Line } \\ \text { FREEDOM } & \text { Following rehabilitation, economics and everyday-dialysis outcome } \\ & \text { measurements } \\ \text { HD } & \text { Hämodialyse } \\ \text { HDF } & \text { Hämodiafiltration } \\ \text { HRQOL } & \text { Health related quality of life } \\ \text { KDOQI } & \text { Kidney disease outcomes quality of life initiative } \\ \text { KDQOL } & \text { Kidney disease quality of life } \\ \text { KfH } & \text { Kuratorium für Dialyse und Transplantation } \\ \text { KI } & \text { Konfidenzintervall } \\ \text { NET } & \text { Nierenersatztherapie } \\ \text { nPNA } & \text { Normalized protein nitrogen appearance } \\ \text { PedsQL } & \text { Pediatric quality of life inventory } \\ \text { PD } & \text { Peritonealdialyse } \\ \text { PEG } & \text { Perkutane endoskopische Gastroenterostomie } \\ \text { UF } & \text { Ultrafiltration } \\ & \end{array}$




\section{Einleitung}

\subsection{Terminale Niereninsuffizienz bei Kindern und Jugendlichen}

\subsubsection{Epidemiologie}

Die Inzidenz der terminalen chronischen Niereninsuffizienz (CNI) ist bei Kindern und Jugendlichen deutlich geringer als bei Erwachsenen. Im europäischen Raum lag die Prävalenz 2015 bei Kindern und Jugendlichen bei 56 pro eine Million altersadjustierter Population. Bei Erwachsenen hingegen bei 810 pro einer Million altersadjustierter Population (1). Weltweit unterscheidet sich die Prävalenz von Kindern und Jugendlichen mit einer Nierenersatztherapie (NET) erheblich: In den entwickelten Ländern ist sie vergleichbar mit den europäischen Daten und in weniger entwickelten Ländern deutlich geringer (2). Unterschiede in der Prävalenz der zugrundeliegenden Erkrankungen spielen hierbei eine untergeordnete Rolle. Vielmehr entscheidend sind die ungenügende Infrastruktur der jeweiligen Gesundheitssysteme und die hohen Kosten, die mit einer NET im Kindes- und Jugendalter verbunden sind. In Schwellenländern steht eine NET, vor allem für jüngere Kinder, häufig nicht zur Verfügung. Vor 1980 wurde auch in Europa, gerade bei kleineren Kindern, häufig keine chronische NET durchgeführt. Im Vergleich zu den 70er Jahren erhöhte sich der relative Anteil von Patient*innen unter zwei Jahren fast um den Faktor 80 in den 80er Jahren (3). Diese Zahlen zeigen die Fortschritte in der Behandlung von Kindern und Jugendlichen mit terminaler CNI.

Allerdings ist die Morbidität und Mortalität der betroffenen Kinder und Jugendlichen erheblich (46). Bei Kindern und Jugendlichen mit NET (Dialyse oder Transplantation) ist die Mortalitätsrate 30-fach höher im Vergleich zur Allgemeinbevölkerung (7). Die Mortalitätsrate ist abhängig von der Art der NET: 2008 lag die jährliche Mortalitätsrate generell bei Kindern bei 0,31 pro 1000 Lebensjahre, wohingegen sie bei Nieren transplantierten Kindern bei 3,5 pro 1000 Lebensjahre und bei Kindern an der Dialyse bei 36 pro 1000 Lebensjahre lag (6). Hauptverantwortlich ist eine ausgeprägte kardiovaskuläre Morbidität (8). Kinder und Jugendliche mit einer NET haben eine signifikant reduzierte gesundheitsbezogene Lebensqualität (Health related quality of life HRQOL) sowohl im Vergleich zu Gesunden als auch im Vergleich zu Kindern und Jugendlichen mit anderen chronischen Erkrankungen wie Diabetes mellitus, kardialen Erkrankungen, schwere Adipositas oder Asthma $(9,10)$.

\subsubsection{Ursachen der terminalen Niereninsuffizienz}

Die Erkrankungen, die zu einer terminalen CNI im Kindes- und Jugendalter führen, unterscheiden sich deutlich vom Krankheitsspektrum bei Erwachsenen (Abbildung 1) 


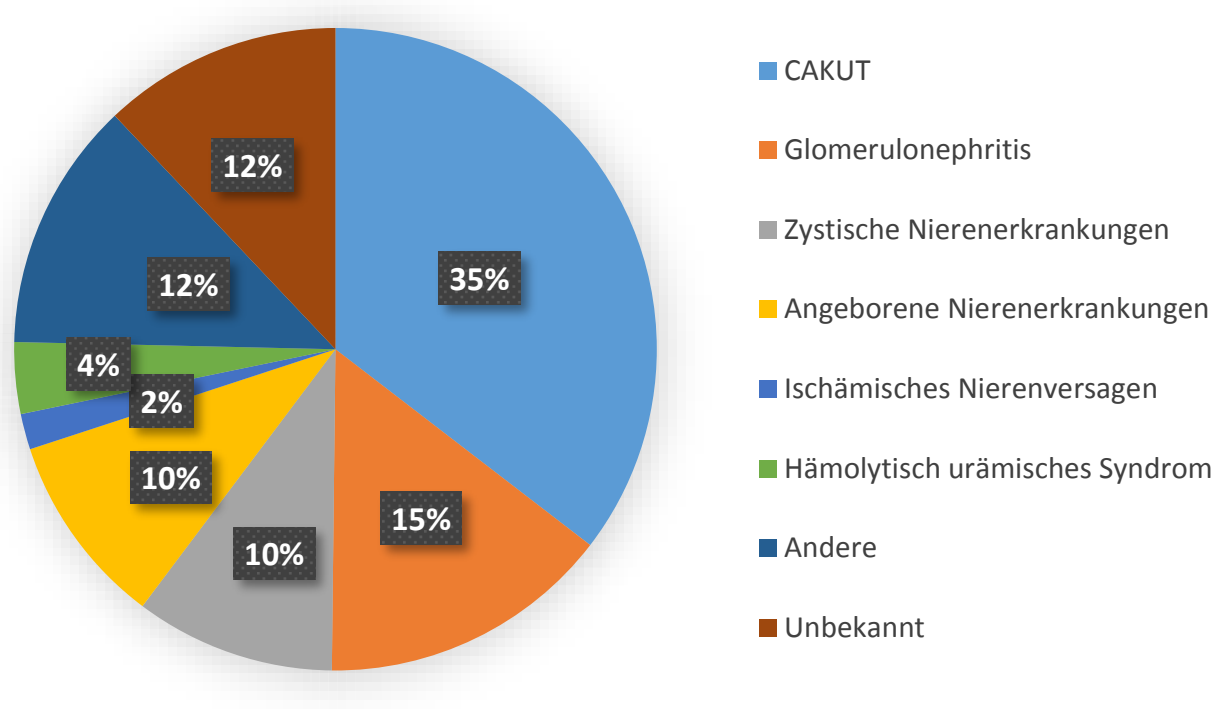

Abbildung 1 Renale Grunderkrankungen von europäischen Kindern und Jugendlichen, die 2015 eine Nierenersatztherapie erhalten haben. Modifiziert nach (1). CAKUT congenital anomalies of the kidney and urinary tract

\section{$\underline{\text { CAKUT }}$}

Die meisten Patient*innen weisen Fehlbildungen im Bereich der Nieren und ableitenden Harnwege auf. Fehlbildungen, die während der Morphogenese der Nieren und/oder ableitenden Harnwege entstehen, werden unter dem Begriff congenital anomalies of the kidney and urinary tract (CAKUT) zusammengefasst (11). Eine klare Definition des Begriffes existiert nicht und die Abgrenzung zu anderen Erkrankungen, wie z.B. Ziliopathien, ist aktuell Gegenstand der Diskussion. Die Prävalenz von CAKUT wird auf zwischen zwei pro 1000 Geburten bis acht pro 1000 Geburten geschätzt (12,13). Damit zählen CAKUT zu den häufigsten angeborenen Fehlbildungen. CAKUT treten isoliert oder als Teil von Syndromen (z.B. branchio-oto-renales Syndrom) auf (14). In den letzten Jahren wurden zahlreiche Gene identifiziert, die CAKUT verursachen können, die Erbgänge sind variabel (15). Mit den 2019 bekannten Genen können 20-25\% der betroffenen Patient*innen molekulargenetisch charakterisiert werden. Diese Gene zeichnen sich durch eine variable Penetranz aus. Der Phänotyp innerhalb einer betroffenen Familie kann von einer isolierten Ureterabgangsstenose bis zur beidseitigen Nierenagenesie reichen (15). Die meisten CAKUT-Patient*innen entwickeln keine oder nur eine gering gradige CNI. Betroffene mit erheblichen Fehlbildungen können bereits ab Geburt oder dem Säuglingsalter eine NET benötigen (16).

\section{Zystische Nierenerkrankungen}

Eine weitere Erkrankungsgruppe, die im Kindes- und Jugendalter zur terminalen CNI führt, stellen die zystischen Nierenerkrankungen dar. Die häufigsten Erkrankungen sind die autosomal- 
rezessive polyzystische Nierendegeneration (autosomal recessive polycystic kidney disease ARPKD), die verschiedenen Formen der Nephronophthise, das Bardet-Biedl-Syndrom und die HNF1-beta-Nephropathie $(17,18)$. Die meisten Gene, die bei zystischen Nierenerkrankungen betroffen sind, kodieren für Proteine im primären Zilium (19) (Abbildung 2). Daher werden diese Erkrankungen auch Ziliopathien genannt. Die Phänotyp-Genotyp-Korrelation ist sehr variabel. So können verschiedene Erkrankungen durch Mutationen in einem Gen verursacht werden. Ebenso können Mutationen in einem Gen sich klinisch in verschiedenen Erkrankungen präsentieren (18). Ziliopathien manifestieren sich häufig auch extrarenal. Bei der ARPKD steht die Leberbeteiligung im Sinne einer periportalen Fibrose oder eines Caroli-Syndroms im Vordergrund (20). Bei der Nephronophthise gibt es zahlreiche extrarenale Manifestationen wie Leberbeteiligung, Entwicklungsdefekte des zentralen Nervensystems, okuläre Manifestationen und Skelettdysplasien (18).

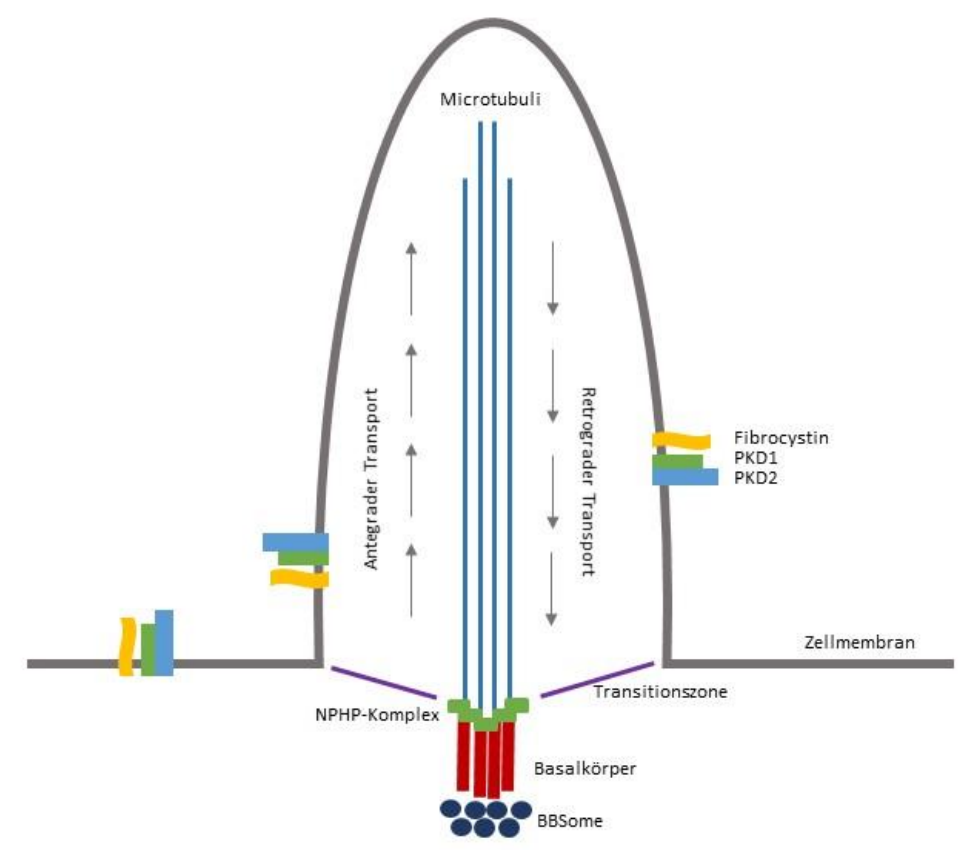

Abbildung 2: Aufbau des Ziliums

Das Zilium unterteilt sich in Basalkörper, Transitionszone, Zilienkörper, Zilienhals und Zilienspitze. Die Transitionszone bildet die Barriere zwischen Zelle und Zilium, über die nur ein selektiver Proteinaustausch stattfinden kann. Zentral im Zilium gelegen sind Microtubuli-Bündel (Axonem) entlang derer der ante- und retrograde intraflagellare Transport stattfindet. Der BBSome-Komplex führt Proteine den Zilien zu, baut im Basalkörper intraflagellare Transportkomplexe zusammen und ist am antegraden Transport in die Zilienspitze beteiligt. Mutationen in BBS Genen führen zum Bardet-Biedl-Syndrom. Der PKD-Komplex (polycystic kidney disease) bestehend aus PKD1, PKD2 und Fibrocystin (PKHD1) ist entlang der Zellmembran renaler Tubuluszellen auch außerhalb des Ziliums lokalisiert. Der Komplex ist in die Zell-Zell-Interaktion involviert und spielt bei der Entwicklung renaler Tubuluszellen eine wichtige Rolle. Mutationen im PKD1 und PKD2 Gen führen zur autosomal-dominanten polyzystischen Nierendegeneration (ADPKD). Mutationen im PKHD1 Gen zur autosomal-rezessiven polyzystischen Nierendegeneration (ARPKD). Die Nephronophthise ist genetisch sehr heterogen. Einige der Nephronophthise Gene, u.a. NPHP1, kodieren für Proteine (NPHP-Komplex) im Bereich der Transitionszone (21). Modifiziert nach (22). 


\section{Glomeruläre Erkrankungen}

Bei den glomerulären Erkrankungen, die im Kindes- und Jugendalter zur terminalen CNI führen, stehen bei Säuglingen und Kleinkindern Steroid-resistente nephrotische Syndrome im Vordergrund. Diese sind häufig verursacht durch Mutationen in Genen, die im Podozyten exprimiert werden (Abbildung 3) (23). Bei Jugendlichen treten zunehmend ähnliche Glomerulonephritiden bzw. Glomerulopathien wie bei Erwachsenen auf: das heterogene Krankheitsbild der fokal-segmentalen Glomerulosklerose, Nierenbeteiligung bei Kollagenosen und Vaskulitiden und die IgA-Nephropathie (16).

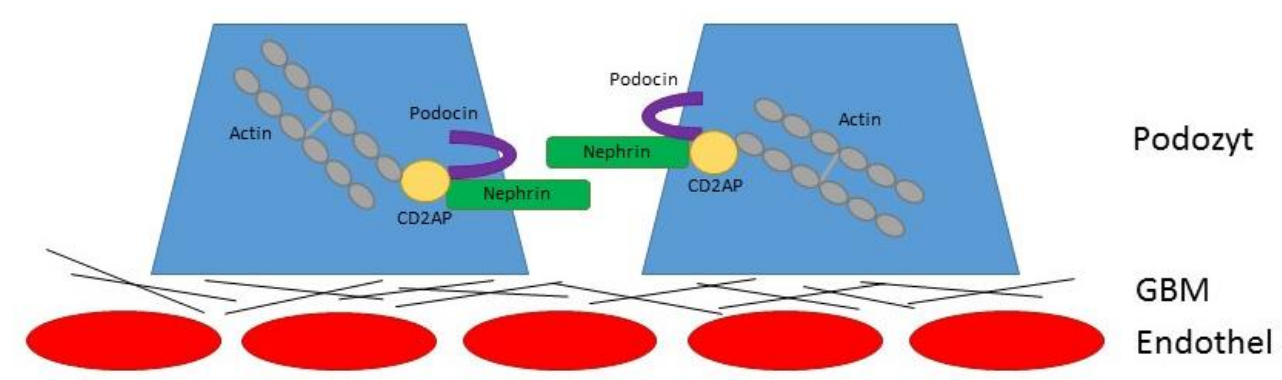

Abbildung 3: Aufbau der glomerulären Filtrationsbarriere

Schematische Darstellung der Schlitzmembran des Podozyten. Nephrin, Podocin und CD2 assoziiertes Protein (CD2AP) bilden einen Komplex, der im Wesentlichen die Filtrationsbarriere des Glomerulus bildet. Mutationen im Nephrin- und Podocin-Gen führen zum früh manifesten Steroid-resistenten nephrotischen Syndrom (24). GBM Glomeruläre Basalmembran.

\subsection{Indikation zum Beginn der Nierenersatztherapie bei Kindern und Jugendlichen}

Spätestens ab einer errechneten glomerulären Filtrationsrate (eGFR) von $30 \mathrm{ml} / \mathrm{min} / 1,73 \mathrm{~m}^{2}$ sollte mit dem oder der Patient*in und der Familie über die verschiedenen NET-Optionen gesprochen werden: Peritonealdialyse (PD), Hämodialyse (HD) und Nierentransplantation (25). Die präemptive Nierentransplantation stellt die NET der Wahl im Kindes- und Jugendalter dar. Meist lässt sich dies in Deutschland aufgrund der Wartezeit nur realisieren, wenn ein oder eine Lebendspender*in zur Verfügung steht. Die Wartezeit auf ein Spenderorgan für Kinder und Jugendliche unter 16 Jahren liegt aktuell bei knapp zwei Jahren. Diese ist im internationalen Vergleich betrachtet relativ lang (26).

Die Dialyse wird bei Kindern und Jugendlichen allgemein als eine überbrückende NET bis zu einer Transplantation gesehen und nicht als endgültige NET. Ziel ist es daher, den oder die Patient*in bereits vor Beginn der Dialyse für eine Transplantation zu evaluieren, um im Idealfall eine präemptive Transplantation zu ermöglichen. 
Die Entscheidung, eine NET bei Kindern und Jugendlichen mit CNI zu beginnen, ist abhängig von mehreren Faktoren (Abbildung 4). Ein wichtiger Faktor stellt die glomeruläre Filtrationsrate (GFR) dar. Das genaueste Verfahren ist hierfür die Berechnung der GFR anhand des Mittelwertes der Kreatinin- und Harnstoff-Clearance aus einem 24 Stunden Sammelurin. Bei Säuglingen und Kleinkinder ist dies nur durch die Anlage eines Blasenkatheters möglich. Eine hinreichende Abschätzung der GFR ist mittels der Schwartz-Formel (eGFR [ml/min/1,73 $\mathrm{m}^{2}$ Körperoberfläche] $=0,413 \times$ Körperlänge $[\mathrm{cm}] /$ Serumkreatinin $[\mathrm{mg} / \mathrm{dl}]$ ) (27) oder anhand des Cystatin CSerumwertes möglich (28).

Nach der 2006 veröffentlichten Leitlinie der National Kidney Foundation Kidney Disease Outcomes Quality Initiative (KDOQI), in der Kinder und Jugendliche explizit erwähnt wurden, stellte eine eGFR unter 8-10 ml/min/1,73 $\mathrm{m}^{2}$ eine absolute Dialyseindikation dar. Eine eGFR zwischen $10-15 \mathrm{ml} / \mathrm{min} / 1,73 \mathrm{~m}^{2}$ galt als eine relative Indikation in Abhängigkeit von klinischen Symptomen (29). Diese Empfehlungen galten sowohl für Kinder und Jugendliche als auch für Erwachsene. Seit 2015 gibt es eine neue KDOQI Leitlinie für Erwachsene, in der Kinder und Jugendliche nicht explizit berücksichtigt sind. In dieser Leitlinie werden die klinischen Symptome als das entscheidende Kriterium für den Beginn einer NET gesehen, unabhängig von der eGFR (25). Die Rationale zu dieser Änderung basieren auf den Ergebnissen der Initiating Dialysis Early and Late (IDEAL) Studie (30). In dieser Studie wurden erwachsene Patient*innen mit CNI in zwei Gruppen unterteilt: Die erste Gruppe sollte eine Dialyse beginnen bei einer eGFR zwischen 10$14 \mathrm{ml} / \mathrm{min} / 1,73 \mathrm{~m}^{2}$, die zweite Gruppe bei einer eGFR zwischen 5-7 ml/min/1,73m². Zwar mussten letztendlich $76 \%$ der Patient*innen aus der zweiten Gruppe die Dialyse aufgrund von klinischen Symptomen früher, bei einer höheren eGFR, beginnen als geplant, dennoch unterschied sich die eGFR bei Beginn der Dialyse zwischen beiden Gruppen signifikant (12 versus 9,8 $\mathrm{ml} / \mathrm{min} / 1,73 \mathrm{~m}^{2}$ ). Im Mittel wurde die Dialyse in der zweiten Gruppe sechs Monate später begonnen. In beiden Gruppen gab es keinen Unterschied im Hinblick auf Mortalität, kardiovaskuläre Ereignisse, Infektionen oder Dialysekomplikationen (30). Für pädiatrische Patient*innen konnte gezeigt werden, dass ein früher Dialysebeginn weder die Hospitalisationsraten noch die linksventrikuläre Masse, als ein Parameter für die kardiovaskuläre Morbidität, senkt (31). Aufgrund dieser Ergebnisse kann daher davon ausgegangen werden, dass Patient*innen von einem frühen, eGFR gesteuerten Dialysebeginn ohne gleichzeitiges Auftreten klinischer Symptome nicht profitieren.

Ein wichtiges Kriterium für die Dringlichkeit einer NET bei Kindern ist eine inadäquate Gewichtsund Längenentwicklung. Aufgrund Urämie induzierter Übelkeit und Erbrechen haben Kinder und Jugendliche mit CNI häufig eine unzureichende Nahrungszufuhr. Wenn trotz intensiver Versuche mittels hochkalorischer Nahrungsmittelsupplementation oral oder über eine Ernährungssonde keine adäquate Gewichtszunahme zu verzeichnen ist, sollte in Zusammenschau mit der eGFR und weiterer Symptome die Indikation zum Beginn einer NET gestellt werden. Die körperliche Leistungsfähigkeit spielt ebenso eine wichtige Rolle bei der Indikation zur Dialyse. Zunehmende Müdigkeit und Einschränkung der körperlichen Leistungsfähigkeit stellen ein wichtiges Kriterium 
dar (32). Die Leistungsfähigkeit ist allerdings bei kleinen Kindern oft schwer zu objektivieren und wird von den Eltern nicht selten überschätzt. Wenn die CNI schon ab dem Säuglingsalter besteht, ist ein Vergleich zur früheren körperlichen Leistungsfähigkeit nicht möglich. Bei älteren Kindern kann als Kriterium die Anzahl der Schulfehltage oder die abnehmende Leistungsfähigkeit im Sportunterricht dienen.

Als absolute Dialyseindikationen gelten die medikamentöse bzw. diätetisch nicht kontrollierbare Hyperkaliämie, Hyperphosphatämie, die schwere metabolische Azidose, sowie die starke Überwässerung und schwere arterielle Hypertonie (29). Komplikationen der Urämie, wie Perikarditis, Kardiomyopathie oder Pankreatitis, stellen ebenfalls absolute Dialyseindikationen dar. Das Behandlungsteam bestehend aus Kindernephrol*innen, Pflegekräften, Psycholog*innen, Sozialarbeiter*innen und Diätassistent*innen sollte über die Indikation zusammen beraten. Die Entscheidung zum Beginn einer NET sollte eine gemeinsame Entscheidung des betroffenen Kindes (je nach Alter), der Eltern und des Behandlungsteams sein.

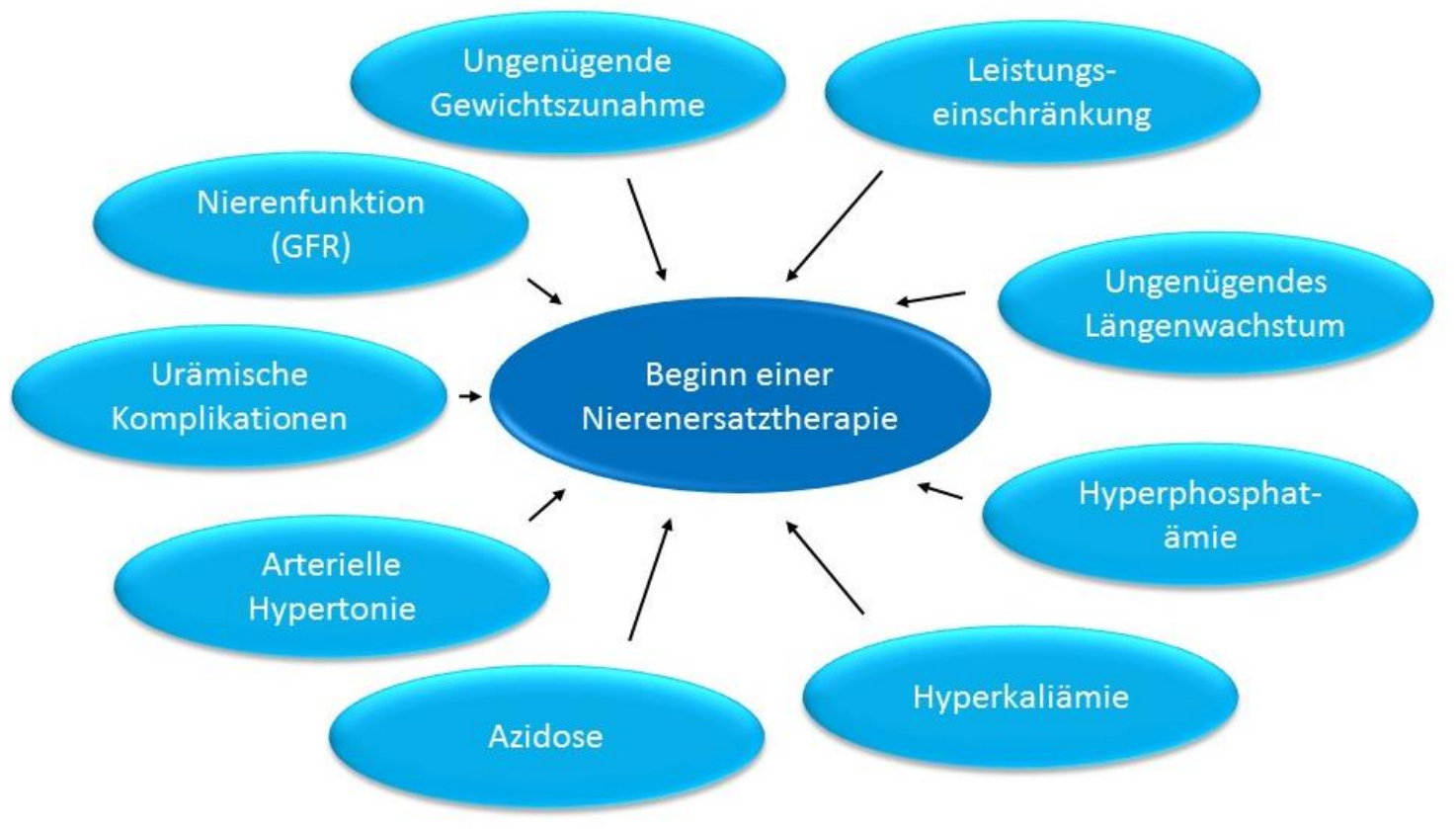

Abbildung 4: Faktoren, die den Beginn einer Nierenersatztherapie bei Kindern und Jugendlichen beeinflussen.

Wenn ein oder eine Lebendspender*in für eine präemptive Transplantation zur Verfügung steht, wird meist die Transplantation vor Beginn der klinischen Symptome bei tendenziell höherer GFR durchgeführt (32).

Dialyse als langfristige Therapieoption oder eine konservative Therapie mit Verzicht einer NET spielen bei Kinder und Jugendlichen mit terminaler CNI eher eine untergeordnete Rolle. Aufgrund der besseren Akutversorgung schwer kranker Früh- und Neugeborener überleben zunehmend Patient*innen mit Multisystemerkrankungen und deutlich verkürzter Lebenserwartung. Wenige entwickeln im weiteren Verlauf eine terminale CNI. Bei diesen Patient*innen, ist die Frage nach 
der individuell besten Therapie (Transplantation, Dialyse als Dauertherapie oder konservative Therapie) oft schwer zu beantworten (33).

Dionne und d'Agincourt-Canning veröffentlichten 2015 eine Empfehlung, welche Aspekte bei der Entscheidung, ob eine NET begonnen werden sollte, berücksichtigt werden sollten (34). Generell empfahlen sie, dass letztendlich das Ziel der Transplantation bei Beginn der Dialyse in Aussicht stehen sollte. So sollte insbesondere bei Früh- und Neugeborenen eine konservative Therapie in Erwägung gezogen werden, wenn es sehr unwahrscheinlich ist, dass der oder die Patient*in diese Zeit bis zu einer möglichen Transplantation überleben wird. Die Lebensqualität des Kindes, die zu erwartende Belastung der Familie und der Wunsch der Familie sollten in die Entscheidung mit einfließen (34).

\subsection{Wahl des Dialyseverfahrens bei Kindern und Jugendlichen}

An Dialysemodalitäten stehen PD und HD als Verfahren mit prinzipiell gleichwertiger Effektivität zur Verfügung (29). Die behandelnden Kindernephrol*innen, Dialysepflegekräfte, die Eltern und der oder die Patient*in sollten gemeinsam entscheiden, welche Dialyseform am besten geeignet ist. Im Vergleich zu Erwachsenen wird im Kindes- und Jugendalter viel häufiger eine PD durchgeführt. In Europa erhielten 2009-2011 47\% der Kinder und Jugendlichen eine PD, 33\% eine HD und $20 \%$ eine Nierentransplantation als erste NET (35).

Die Wahl der Dialysemodalität ist meist altersabhängig (35). Bei Säuglingen und jüngeren Kindern wird meist PD als NET gewählt. Die PD ermöglicht die Therapie zu Hause und erspart den Kindern und ihren Familien den häufigen Weg zum Dialysezentrum. Die PD erfolgt meist in den vergleichsweise langen Schlafphasen und ist deshalb gut mit Kindergarten, Schule und Sozialleben kompatibel (Tabelle 1).

\begin{tabular}{|cll|}
\hline \multicolumn{1}{c}{ Vorteile } & \multicolumn{1}{c|}{ Nachteile } \\
\hline HD & - Dialyse nur alle 2-3 Tage & - Verlust der Restdiurese \\
& - Weniger medizinische & - Systemische Antikoagulation \\
& Verantwortung für die & - Zeitliche und örtliche Abhängigkeit \\
& Patient*innen und Eltern & \\
\hline mit Katheter & - Keine Punktionsschmerzen & - Infektionsgefahr \\
& & - Kein Schwimmen/Baden \\
\hline mit AV-Fistel & - Kein Fremdkörper & - Punktionsschmerzen \\
& - Geringe Infektionsgefahr & \\
& - Sport und Schwimmen fast & \\
& uneingeschränkt möglich & \\
\hline PD & - Keine Punktionsschmerzen & - Große Verantwortung für die \\
& - Längerer Erhalt der Restdiurese & Betroffenen \\
& - Zu Hause durchführbar & - Infektionsgefahr \\
& - Zeitliche Flexibilität & - Baden sehr eingeschränkt \\
& & - Tägliche Dialyse \\
\hline
\end{tabular}

Tabelle 1 Vor- und Nachteile der Hämodialyse (HD) und der Peritonealdialyse (PD) im Kindesund Jugendalter 
Ab dem Schulalter richtet sich die Wahl nach der Präferenz des oder der Patient*in und der Familie (Tabelle 1). Jugendliche bevorzugen häufiger die HD über eine AV-Fistel, da diese mit kürzeren Dialysezeiten und meist ohne Fremdkörper (Katheter) einhergeht.

\subsubsection{Peritonealdialyse}

Vor allem bei Säuglingen, Kleinkindern und Kindern im Grundschulalter wird die PD gegenüber der HD bevorzugt. Da die PD keinen extrakorporalen Kreislauf fordert, führt sie seltener zu kardiovaskulärer Instabilität als die HD. Die renale Restfunktion wird mit PD besser erhalten als mit HD. Daher eignet sich die PD vor allem als erste NET gut, wenn viele Patient*innen noch eine Restdiurese aufweisen (36).

Die PD kann zu Hause durchgeführt werden und wird meist automatisiert, intermittierend über Nacht durchgeführt. Hierfür bieten verschiedene Hersteller Cycler mit kinderadaptierten Systemen an. Eine kontinuierliche ambulante Peritonealdialyse (CAPD) ist ebenfalls möglich. Der Vorteil der automatisierten, nächtlichen PD besteht darin, dass die Kinder tagsüber eine Kindertagesstätte oder Schule besuchen können. Da nur einmal pro Tag eine Konnektion bzw. Diskonnektion durchgeführt wird, ist das Risiko für eine PD-assoziierte Peritonitis geringer als bei der CAPD (37). Allerdings sind übliche Dialysezeiten bei der automatisierten, nächtlichen PD 1012 Stunden, so dass die PD für ältere Schulkinder die Freizeitgestaltung erheblich einschränken kann.

Die PD stellt für den oder die Patient*in und für die Familie eine große Herausforderung dar (38). Sie verlangt ein hohes Maß an Motivation, Organisation und Disziplin. Vor Beginn der PD sollten die psychosoziale Situation und die Wohnverhältnisse der Familie evaluiert werden. Mindestens zwei Bezugspersonen sollten mit der Handhabung der Dialyse vertraut sein. Jederzeit muss ein Kontakt mit dem betreuenden Dialysezentrum gewährleistet sein. Da die PD fast immer über Nacht durchgeführt wird, ist der Schlaf aller beteiligten Personen häufig durch Alarme des Cyclers unterbrochen. Zudem erfolgt die Ernährung von dialysepflichtigen Säuglingen und Kleinkindern meist über eine PEG-Sonde mittels Pumpe über Nacht, was den Schlaf zusätzlich stören kann. Da die PD für die Familien eine große zeitliche und psychische Belastung darstellen kann, sollte Innen Unterstützung von Seiten des Dialysezentrums und ggf. des Jugendamtes (z. B. Familienhilfe) angeboten werden (38). Eine Assistenz durch einen Pflegedienst ist für viele Familien ebenfalls eine große Erleichterung.

Bei Kindern und Jugendlichen ist es häufig möglich, bis zur Transplantation eine PD durchzuführen. Gründe für den Wechsel zur HD sind der Wunsch des oder der Patient*in, schwere, rezidivierende Peritonitiden oder inadäquate Dialyse aufgrund des Versagens des Peritoneums als Dialysemembran (39).

Im Mittel werden Kinder und Jugendliche nach zwei Jahren in Deutschland Nieren transplantiert. Wenn die Dialysezeit z.B. aufgrund von seltenen HLA-Loci oder Sensibilisierungen deutlich 
länger wird, sollte über einen Wechsel zur HD nachgedacht werden. Insbesondere, wenn die Sekundärerkrankungen schlecht beherrschbar sind. In der längerdauernden Dialyse (>2 Jahre) sind die Mortalitätsraten für Kinder und Jugendliche unter PD höher als unter HD (40).

\subsubsection{Hämodialyse}

Wenn HD als NET geplant ist, sollte bei einer GFR um $15 \mathrm{ml} / \mathrm{min} / 1,73 \mathrm{~m}^{2}$ die Anlage einer arteriovenösen Fistel (AV-Fistel) erfolgen. Empfohlen wird dies für alle Kinder ab dem Schulalter und für Jugendliche, falls keine zeitnahe Lebendspende geplant ist (25). Trotz dieser Empfehlung zur Fistelanlage werden ca. $60 \%$ der Kinder und Jugendlichen in Europa über einen zentralvenösen Katheter dialysiert (41). Zwischen den einzelnen Zentren gibt es erhebliche Unterschiede. Dies ist auf das Alter der Patienten, den Gefäßstatus, die Erfahrungen des Zentrums und die Expertise der jeweiligen Gefäßchirurg*innen zurückzuführen (41). Die AVFistelanlage und spätere Benutzung setzt voraus, dass das Kind über mehrere Stunden weitestgehend ruhig sitzt oder liegt und die Shuntpunktion toleriert. Die AV-Fistel sollte mindestens vier bis sechs Wochen reifen. Bei früherer Punktion gibt es bei Kinder und Jugendlichen häufiger Shuntprobleme wie Thrombosen (42). Bei einem notwendigen sofortigen Dialysebeginn sollte daher parallel zur AV-Fistel ein zentralvenöser Dialysekatheter angelegt werden.

Bei jüngeren Kindern erfolgt die HD über zentralvenöse Dialysekatheter. Zentralvenöse Katheter zeigen im Vergleich zu AV-Fisteln signifikant höhere Infektions-, Thrombosierungs- und Fehlfunktionsraten auf, so dass häufiger Revisionen notwendig sind $(41,42)$. Die Blutflussraten sind gerade bei älteren Kindern und Jugendlichen über eine AV-Fistel häufig deutlich besser als über einen zentralvenösen Katheter. Die HRQOL der mit AV-Fistel versorgten Kinder ist vergleichbar mit denen, die über einen zentralvenösen Katheter dialysiert werden (43).

Säuglinge und Kleinkinder erhalten meist eine PD, allerdings kann auch in dieser Altersgruppe eine HD erfolgen. Meist erfolgt dies, wenn Kontraindikationen für eine PD vorliegen (z.B. schwere abdominale Verwachsungen, rezidivierende Peritonitiden) oder eine PD von Seiten des häuslichen Umfeldes nicht durchgeführt werden kann. Aufgrund der in diesem Alter relativ hohen Flüssigkeitszufuhr und der schlechteren Toleranz der Ultrafiltration (UF) sind oft vier und mehr HD-Behandlungen pro Woche erforderlich. Der Betreuungsaufwand während der HD ist in dieser Altersgruppe enorm und erfordert häufig ein pflegerische Einzelbetreuung während der Dialysesitzung (44). 


\subsection{Messung der Dialysequalität}

\subsubsection{Kt/V}

Ein wesentliches Problem jeglicher Dialyse stellt die Messung dar, wie gut eine Dialyse ist. Im angloamerikanischen hat sich der Begriff dialysis adequacy etabliert. Im Deutschen wird hierfür der Begriff Dialysequalität verwendet. Ein Maß für die Dialysequalität ist die Elimination (Clearance) kleinmolekularer Substanzen. Als ein Parameter für die Effektivität einer Dialyse wurde $\mathrm{Kt} / \mathrm{V}$ eingeführt. Das von Harnstoff gereinigte Blutvolumen wird ins Verhältnis zum Harnstoff-Verteilungsvolumen gesetzt. In der Formel von Daugirdas wird zusätzlich der Harnstoff berücksichtigt, der während der Dialyse vom Körper gebildet wird, und der Harnstoff, der durch UF dem Körper entzogen wird (45). Bei der HD wird das Kt/V für eine einzelne Dialysesitzung berechnet. Es sollte mindestens ein Kt/V von 1,2 pro Sitzung erreicht werden (29).

Für die PD wurde diese Formel adaptiert. Sie berücksichtigt die Harnstoff-Clearance und das Harnstoff-Verteilungsvolumen und wird auf eine Woche bezogen (46). Die Zielwerte liegen bei mindestens 1,7 (29). Bei vorhandener Restdiurese kann die Eliminationsleistung der Niere als Nieren-Kt/V einberechnet werden (47).

Die Kt/V Zielwerte für HD und PD orientieren sich an Daten, die anhand von Studien mit erwachsenen Dialysepatient*innen erhoben wurden (29). Für Kinder und Jugendliche wurden diese Zielwerte nicht evaluiert. Ein Zusammenhang zwischen Höhe des Kt/V und der Mortalität, wie es für erwachsene Dialysepatient*innen gezeigt werden konnte (48), konnte bei pädiatrischen Dialysepatient*innen nicht bestätigt werden (49).

\subsubsection{Renale Restfunktion}

Die renale Restfunktion ist ein entscheidender Faktor, um die Sekundärerkrankungen der CNI an der Dialyse besser zu beherrschen. Daher stellt der möglichst lange Erhalt der renalen Restfunktion ein $\mathrm{Maß}$ für die Dialysequalität dar. Durch die renale Restfunktion wird der Flüssigkeits- und Natriumhaushalt besser kontrolliert. Die Volumenschwankungen zwischen den einzelnen Dialysesitzungen sind geringer und die Flüssigkeitsüberladung im Dialyse-freien Intervall wird weniger. Daher ist der arterielle Blutdruck meist besser kontrollierbar (50). Die Elimination von Kalium und Phosphat über die Nieren ermöglicht den Dialysepatient*innen eine freiere Kostaufnahme (51). Die weniger strikten diätetischen Einschränkungen und die freiere Flüssigkeitsaufnahme tragen sicherlich dazu bei, dass die Lebensqualität von Patient*innen mit erhaltener Restdiurese im Vergleich zu anurischen Dialysepatienten*innen besser ist (50). Neben der glomerulären UF werden über die Niere Protein gebundene Toxine eliminiert. Diese tubuläre Sekretion lässt sich über eine Dialyse nicht adäquat ersetzen $(52,53)$. Sie stellt somit einen weiteren, entscheidenden Vorteil der renalen Restfunktion dar. Die chronische Inflammation, die 
bei Patient*innen mit CNI zu beobachten ist, und die urämische Vaskulopathie sind weniger ausgeprägt (54), so dass adulte Dialysepatient*innen mit erhaltener Diurese eine geringere Mortalität aufweisen $(51,55)$. Der Verlust der renalen Restfunktion kann durch Verzicht auf nephrotoxische Substanzen (vor allem Medikamente) und geringere UF-Raten minimiert werden (36). Bei der PD sind die UF-Raten geringer als bei der HD. Dies trägt dazu bei, dass die renale Restfunktion mit PD besser erhalten bleibt (36).

\subsubsection{Klinische Symptome}

Klinische Symptome sind meist durch eine unzureichende Kontrolle der Folgen der CNI verursacht. Symptome wie Müdigkeit, Juckreiz und Schlafstörungen beinträchtigen den Alltag der Dialysepatient*innen erheblich (56,57). Neben einer unzureichenden Gewichts- und Längenentwicklung sind Müdigkeit, eingeschränkte kognitive und körperliche Leistungsfähigkeit die wichtigsten Symptome, um die Dialysequalität bei Kindern und Jugendlichen zu beurteilen (58). Den Kindern und Jugendlichen sollte es möglich sein, eine Schul- und spätere Berufsausbildung zu absolvieren. In der Dialyse-freien Zeit sollte es den Betroffenen möglich sein, ihre sozialen Kontakte zu pflegen und Freizeitaktivitäten nachzugehen.

\subsection{Qualität der Dialyseverfahren}

Bei Dialysepatient*innen ist häufig die Calcium-Phosphat-Homöostase weitreichend gestört. Folge hiervon sind die renale Osteopathie und urämische Vaskulopathie $(59,60)$. Dialysepatient*innen befinden sich in einem Zustand der chronischen Inflammation ausgelöst durch mangelnde renale Ausscheidung von Zytokinen, immunologische Dysregulation und direkte immunologische Reaktion auf Bestandteile der Dialyse wie Dialysewasser und Dialysemembranen (61). Die chronische Inflammation begünstigt vaskuläre Umbauprozesse. Die urämische Vaskulopathie, die urämische Kardiomyopathie und arterielle Hypertonie sind hauptverantwortlich für die hohe Mortalität der Dialysepatient*innen (62). Auch pädiatrische Dialysepatient*innen weisen bereits eine urämische Vaskulopathie auf $(63,64)$. Der arterielle Blutdruck kann oft trotz intensiver medikamentöser Therapie nur unzureichend eingestellt werden (65). Kramer et al. analysierten wie viele Kinder und Jugendliche mit Dialysebehandlung eine unkontrollierte arterielle Hypertonie (arterieller Blutdruck über der 95\% Körperlängenentsprechenden Perzentile unabhängig von der Anzahl der Antihypertensiva) aufwiesen. Sie untersuchten europaweit 464 pädiatrische HD- und 851 PD-Patient*innen. 46\% der HD- und 36\% der PD-Patient*innen hatten eine unkontrollierte arterielle Hypertonie (66).

Funktionelle Gefäßveränderungen und strukturelle Gefäßumbauten können non-invasiv untersucht werden (z.B. mittels Pulswellengeschwindigkeit, Intima-Media-Dicke oder Computertomographie der Koronargefäße). Civilibal et al. untersuchten 53 Kinder und Jugendliche mittels Computertomografie der Koronargefäße. 15\% der Patient*innen mit einem 
medianen Alter von 16,4 Jahren wiesen koronare Verkalkungen auf (67). Die Patientinnen mit koronaren Verkalkungen waren signifikant länger an der Dialyse, wiesen höhere Phosphatserumwerte und Parathormonspiegel auf im Vergleich zu Patient*innen ohne koronare Verkalkungen (67). Covic et al. untersuchten die aortale Pulswellengeschwindigkeit als Maß für die Gefäßsteifigkeit bei pädiatrischen HD-Patient*innen. Die aortale Pulswellengeschwindigkeit war signifikant höher bei HD-Patient*innen im Vergleich zu gleichaltrigen gesunden Kontrollpersonen (68). Eine erhöhte arterielle Gefäßsteifigkeit war bei Erwachsenen mit einer erhöhten Mortalität assoziiert (69).

Aufgrund von kognitiver und körperlicher Leistungseinschränkungen können viele Dialysepatient*innen nur begrenzt am Alltagsleben teilnehmen. Die Partizipation an Aktivitäten des täglichen Lebens ist deutlich reduziert (70). Diese Einschränkung lässt sich gleichermaßen bei HD- und bei PD-Patient*innen beobachten (70). Die Berufsausbildung bzw. Ausübung des Berufes ist fast immer unmöglich (71). Dialysepatient*innen sind signifikant weniger körperlich aktiv als Gleichaltrige, die keine terminale CNI haben (56). Murtagh et al. analysierten, dass $71 \%$ erwachsener Dialysepatient*innen über Müdigkeit/Fatigue, 55\% über Pruritus und 44\% über Schlafstörungen berichteten (57). Müdigkeit und depressive Symptome traten bei Dialysepatient*innen vergleichbar häufig wie bei Patient*innen mit einer malignen, hämatologischen Grunderkrankung unter Chemotherapie auf (72). Die Patient*innen müssen diätetische Vorschriften einhalten und dürfen oft nur eingeschränkt Flüssigkeit zu sich nehmen. Diese diätetischen Restriktionen belasten den Alltag der Dialysepatient ${ }^{*}$ innen oft erheblich.

Pädiatrische Dialysepatient*innen zeigten ebenfalls eine signifikante Beeinträchtigung der HRQOL, vor allem in den Bereichen Kognition, Schmerzen und Emotionen (73). Sie sind häufig hospitalisiert. Das Wachstum von Kindern und Jugendlichen, die eine Dialyse erhalten, ist beeinträchtigt. Die Hauptursachen hierfür sind unzureichende Nahrungsaufnahme, metabolische Azidose, sekundärer Hyperparathyreoidismus und eine Wachstumshormonresistenz bei CNI (74). Auch nach erfolgreicher Nierentransplantation kommt es häufig nicht zu einem kompletten Aufholwachstum, so dass fast die Hälfte der Erwachsenen, die als Kind eine Dialyse erhalten haben, eine Endgröße unter der 3. Perzentile aufweisen (75).

Die hier beschriebene erhebliche Morbidität prävalenter Dialysepatient ${ }^{\star}$ innen ist die Motivation, Strategien zur Verbesserung der Dialysequalität zu etablieren.

\subsection{Ansätze zur Verbesserung der Dialysequalität}

Die theoretische Grundlage bildet die Annahme, dass es sowohl zwischen der Dialysezeit und der Überlebenswahrscheinlichkeit als auch zwischen dem $\mathrm{Kt} / \mathrm{V}$ und der Überlebenswahrscheinlichkeit einen exponentiellen Zusammenhang gibt (siehe Abbildung 5) (76). 

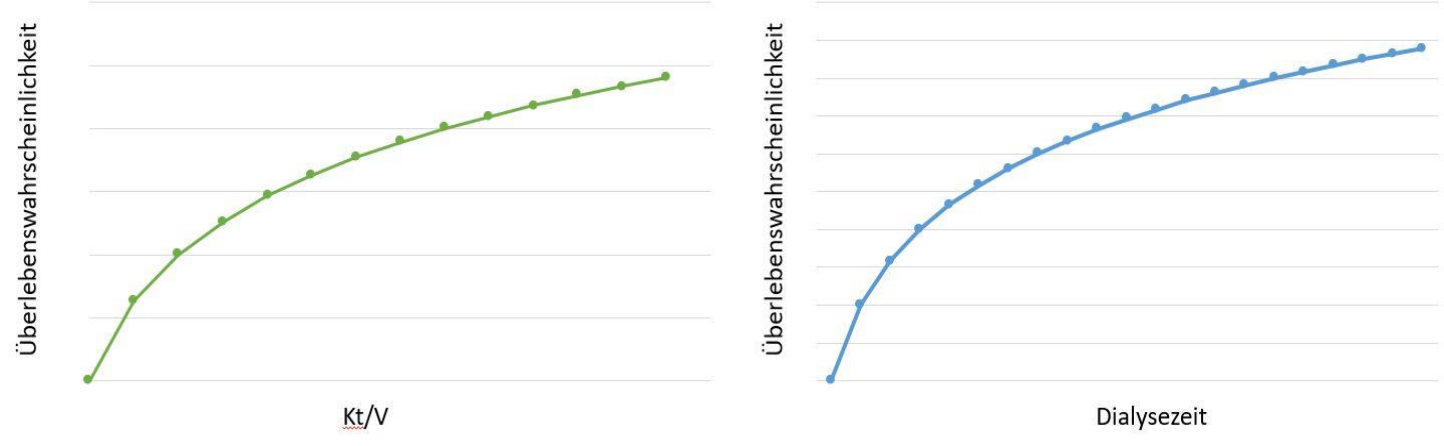

Abbildung 5 Hypothetischer Zusammenhang zwischen Überlebenswahrscheinlichkeit und KT/V bzw. Dialysezeit

Die $x$ - und y-Achse sind nicht skaliert, da der exakte Kurvenverlauf nicht bekannt ist. Mit höherem $\mathrm{Kt} / \mathrm{V}$ und längerer Dialysezeit verbessert sich die Überlebenswahrscheinlichkeit zunächst deutlich bis die Überlebenswahrscheinlichkeit nahezu parallel zur x-Achse verläuft. Bei anurischen Patient ${ }^{*}$ innen ist die Überlebenswahrscheinlichkeit $0 \%$ bei einem $\mathrm{Kt} / \mathrm{V}$ von 0 oder einer Dialysezeit von 0 . Modifiziert nach (76).

\subsubsection{Erhöhung Kt/V}

Sowohl für die PD als auch die HD wurden prospektive, kontrollierte Studien durchgeführt, die untersuchten, ob eine erhöhte Elimination kleinmolekularer Substanzen die Mortalität von Dialysepatient*innen senkt $(77,78)$. In der ADEMEX-Studie (Adequacy of Peritoneal Dialysis in Mexico) erreichten die 484 PD-Patient*innen der Kontrollgruppe ein mittleres wöchentliches Kt/V von 1,62. Bei den 481 Patient*innen der Interventionsgruppe lag das $\mathrm{Kt} / \mathrm{V}$ am Studienende signifikant höher bei 2,13. Die 2-Jahres-Überlebenswahrscheinlichkeiten waren in beiden Gruppen gleich: in der Kontrollgruppe bei 68,3\% (95\%-Konfidenzintervall (KI) 64,2-72,9\%) und in der Interventionsgruppe bei 69,3\% (95\%-KI 65,1-73,6\%) (77).

In der HEMO-Studie (hemodialysis study) wurde in einem 2x2-faktoriellen Design sowohl der Einfluss eines Standard Kt/V versus erhöhtem Kt/V als auch der Einfluss von low-flux versus highflux Dialysatoren (Dialysefiltern) untersucht (78). Die 926 Patient*innen der Kontrollgruppe (Kt/V 1,32) unterschieden sich nicht in der Mortalitätsrate, dem primären Endpunkt, von den 920 Patient*innen mit erhöhter Dialysedosis $(K t / V$ 1,71). Das relative Mortalitätsrisiko (Gruppe mit erhöhtem Kt/V versus Kontrollgruppe) lag bei 0,96 (95\%-KI 0,84-1,10) (78).

\subsubsection{High-Flux-Dialysator}

Die HD kann durch die Verwendung von Dialysatoren mit einem höheren UF-Koeffizienten modifiziert werden. Der UF-Koeffizient gibt an, wieviel $\mathrm{ml}$ UF pro Stunde pro $\mathrm{mmHg}$ Transmembrandruck erreicht werden kann. Dialysatoren mit einem UF-Koeffizienten >20 $\mathrm{ml} / \mathrm{h} / \mathrm{mmHg}$ werden als high-flux Dialysatoren bezeichnet. Die hohe hydraulische Permeabilität 
der Membran ermöglicht zusätzlich eine Elimination mittels Filtration von Mittelmolekülen (Substanzen mit einem Molekulargewicht von 500-15.000 Dalton; z.B. $\beta 2-M i k r o g l o b u l i n)$ (79).

In der HEMO-Studie erreichten die Patient*innen, die mit einem low-flux Dialysator behandelt wurden, eine mittlere $\beta 2$-Mikroglobulin-Elimination von $3,4 \mathrm{ml} / \mathrm{min}$. Die Patient*innen, die mit

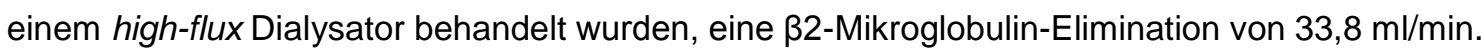
Jedoch zeigte sich bei beiden Gruppen kein Unterschied in der Mortalität, dem primären Endpunkt der Studie (Relatives Mortalitätsrisiko: high-flux Gruppe versus low-flux Gruppe 0,92; 95\%-KI $0,81-1,05)(78)$.

\subsubsection{Hämodiafiltration}

Ein weiterer Ansatz ist die Hämodiafiltration (HDF) eine Kombination aus Hämodialyse und Hämofiltration. Die Entfernung von harnpflichtigen Substanzen wird hierbei sowohl durch Diffusion (Dialyse) als auch durch Konvektion (Filtration) erreicht. Die für die Filtration erforderliche Substitutionslösung stammt aus vorgefertigten Beuteln oder wird von der Dialysemaschine unter Verwendung des Dialysates produziert. Das letztere Verfahren wird online HDF genannt (79). Durch den zusätzlichen konvektiven Stofftransport werden Mittelmoleküle besser eliminiert (80). Die Effektivität einer HDF-Behandlung lässt sich mit Hilfe des Volumens, das in einer Sitzung substituiert wird, abschätzen. Im Dialysestandard der Deutschen Gesellschaft für Nephrologie (DGfN) wird ein Mindestvolumen von 2,5 Litern pro Stunde gefordert (81).

In den letzten Jahren wurden drei prospektive, randomisierte Studien bei Erwachsenen durchgeführt, die die Mortalität mit HDF im Vergleich zur HD untersuchten. In der CONTRASTStudie (CONvective TRAnsport Study) wurden 356 HD-Patient*innen mit 358 HDF-Patient ${ }^{*}$ innen prospektiv verglichen (82). Nach einer mittleren Beobachtungsdauer von drei Jahren zeigte sich kein Unterschied zwischen der HD-Gruppe und der HDF-Gruppe hinsichtlich der Mortalität (hazard ratio 0,95; 95\%-KI 0,75-1,2) und der Anzahl kardiovaskulärer Ereignisse (hazard ratio $1,07 ; 95 \%-K I 0,83-1,39)(82)$.

In der türkischen online HDF Studie wurde als primärer kombinierter Endpunkt die Mortalität und die Anzahl nicht-tödlicher kardialer Ereignisse gewählt. Nach einer mittleren Beobachtungsdauer von zwei Jahren unterschieden sich die HD-Patient*innen $(n=391)$ nicht von den HDFPatient*innen $(n=391)$ hinsichtlich des Erreichens des primären Endpunktes (hazard ratio 0,82; 95\%-KI 0,59-1,16) (83). In einer post hoc Analyse zeigten HDF Patient*innen, die mit einem mittleren Substitutionsvolumen von $>17,4$ Litern pro HDF-Sitzung behandelt wurden, ein verbessertes Überleben (83).

In der ESHOL-Studie (Estudio de Supervivencia de Hemodiafiltración On-Line) zeigte sich bei HDF-Patient*innen $(n=456)$ eine signifikant geringere Mortalitätsrate im Vergleich zu HDPatient*innen $(n=450)$ (hazard ratio 0,70; 95\%-KI 0,53-0,92) (84). Hypotensive Episoden traten 
signifikant weniger mit HDF im Vergleich zur HD auf (84). Dies spricht für eine bessere hämodynamische Stabilität bei der HDF-Behandlung.

Eine Metanalyse kontrollierter, randomisierter Studien zur HDF zeigte keinen signifikanten Unterschied in der Gesamt-Mortalität. Die Mortalität aufgrund kardiovaskulärer Ereignisse war schwach signifikant geringer unter HDF im Vergleich zur HD (85).

Die Ergebnisse einer multizentrischen, nicht-randomisierten Studie bei pädiatrischen Dialysepatient*innen, die die Effekte der HDF auf kardiovaskuläre Parameter untersuchte, sind noch nicht publiziert (86).

\subsubsection{Dialysezeit}

Die konventionelle HD wird meist an drei bis vier Tagen die Woche über jeweils vier bis fünf Stunden durchgeführt. Somit liegt die wöchentliche Gesamtdialysezeit bei ca. 12-15 Stunden. Um die Dialysequalität zu steigern, kann auch die Dialysezeit verlängert werden.

In der DOPPS (Dialysis Outcomes and Practice Pattern Study) Studie konnte gezeigt werden, dass eine Verlängerung der Dialysesitzung über vier Stunden die Mortalität bei erwachsenen HDPatient*innen signifikant senkte (48). 1980 HD-Patient*innen hatten Dialysesitzungen über vier Stunden. Ihr relatives Mortalitätsrisiko in Bezug auf die 14353 HD-Patient*innen mit Dialysesitzungen weniger als vier Stunden lag mit 0,73 signifikant niedriger. Jede Verlängerung der Dialysesitzung um jeweils 30 Minuten, ging mit einem 7\% niedrigeren Mortalitätsrisiko einher (48).

\subsection{Intensivierte Hämodialyse-Verfahren}

\subsubsection{Definition und Modalitäten}

Als intensivierte HD-Verfahren werden Dialyseverfahren mit einer verlängerten Gesamtdialysezeit bezeichnet. Da bereits die konventionelle HD keine genaue Zeitdefinition enthält, ist die Definition der intensivierten HD ebenfalls nicht einheitlich. Meist wird eine Gesamtdialysezeit ( $\geq 15$ Stunden/Woche) als intensiviert bezeichnet.

Eine verlängerte Dialysezeit kann über eine Verlängerung der einzelnen Dialysesitzungen (mehr als fünf Stunden) und/oder eine höhere Frequenz der Dialysesitzungen (mehr als vier pro Woche) erreicht werden. In der Praxis haben sich hauptsächlich drei Modalitäten etabliert: Kurze tägliche HD (zwei bis drei Stunden an fünf bis sieben Tagen pro Woche), nächtlich intermittierende HD (sechs bis acht Stunden an drei Tagen pro Woche) oder täglich nächtliche HD (sechs bis acht Stunden an fünf bis sieben Tagen pro Woche) (87). Allerdings ist die Bezeichnung "häufig“ anstelle von „täglich“ zutreffender, da die Verfahren meist an fünf bis max. sechs Tagen die 
Woche durchgeführt werden. Da in der Literatur diese „häufigen“ intensivierten Verfahren fast immer mit „täglich“ bezeichnet werden, wird dieser Begriff im Folgenden verwendet.

Jedes der Verfahren kann entweder zu Hause oder in einem Dialysezentrum durchgeführt werden. Die nächtlichen Verfahren werden meist zu Hause oder im Dialysezentrum unter limited care Bedingungen durchgeführt. Limited care bedeutet, dass der Dialysearzt oder die Dialyseärztin nicht vor Ort ist, aber in ständiger Rufbereitschaft zur Behandlung möglicher akuter Notfälle. Mindestens einmal pro Woche erfolgt eine ärztliche Visite (81). Dieses Verfahren wird auch als zentralisierte Heimdialyse bezeichnet.

Bei den nächtlichen Verfahren mit langer Sitzungsdauer kann der Blut- und Dialysatfluss reduziert werden, um eine zu hohe Elimination z.B. von Phosphat zu verhindern. Eine weitere Möglichkeit stellt die Kombination der langen Sitzungsdauer mit HDF dar. Letztendlich kann je nach örtlichen Begebenheiten und dem Wunsch des oder der Patient*in ein individuelles, intensiviertes HDProgramm zusammengestellt werden (siehe Abbildung 6).

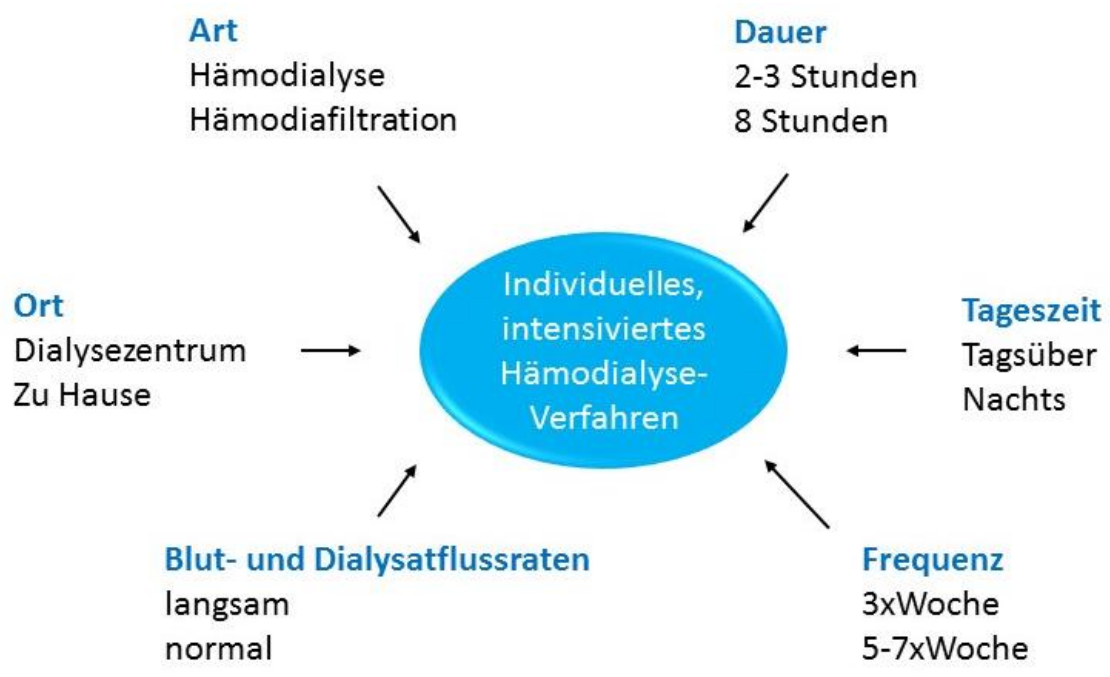

Abbildung 6: Möglichkeiten des individuellen, intensivierten HD-Programms. Modifiziert nach (88).

\subsubsection{Rationale intensivierter Hämodialyseverfahren}

Intensivierte HD-Verfahren erhöhen das Kt/V deutlich (89). Zusätzlich verbessert sich die Elimination der Mittelmoleküle unter intensivierter HD (90). Aufgrund der längeren Dialysezeit sind die UF-Raten pro Stunde bei intensivierter HD geringer als bei konventioneller HD und somit weniger belastend für den Kreislauf. Patient*innen mit geringerer UF-Rate erholen sich schneller nach der Dialysesitzung (91). 
Hohe UF-Raten sind mit einer erhöhten Mortalität assoziiert (92-94). In einer großen, retrospektiven Studie mit 118394 HD-Patient*innen hatten Patient*innen mit einer mittleren UFRate von über $13 \mathrm{ml} / \mathrm{kg} / \mathrm{h}$ eine $30 \%$ höhere Mortalitätsrate als Patient*innen mit geringerer UFRate (hazard ratio 1,31; 95\%-KI 1,28-1,34) (94). Neben hohen UF-Raten ist eine hohe prädialytische Flüssigkeitsüberladung mit einer erhöhten Mortalität assoziiert (95). Die hohe prädialytische Flüssigkeitsüberladung lässt sich vor allem mit häufigeren Sitzungen pro Woche reduzieren.

\subsubsection{Intensivierte Hämodialyseverfahren bei Erwachsenen}

Erste Berichte über intensivierte HD stammen von Guy Laurent aus Tassin, Frankreich (96). Dort wurden ab den 60er Jahren Patient*innen über 24-30 Stunden pro Woche hämodialysiert. Keiner der Patient ${ }^{*}$ innen benötigte mit dieser intensivierten HD eine antihypertensive Therapie. Die 10Jahresüberlebensrate lag bei $85 \%$ (96). Seit Ende der 90er Jahre gibt es zunehmend Berichte über Fallserien und retrospektive Studien zur intensivierten HD.

Vier randomisierte, prospektive, kontrollierte Studien, die die Effekte intensivierter HD untersuchten, sind inzwischen publiziert worden (97-100):

\section{Short Daily Trial}

Im Short Daily Trial der The Frequent Hemodialysis Trial Group wurden Patient*innen ( $n=120)$, die mit konventioneller (drei Mal pro Woche) HD $(n=120)$ behandelt wurden, mit Patient*innen verglichen, die sechs Mal pro Woche dialysiert wurden $(n=125)$ (97). Die HD fanden im Dialysezentrum statt. Als Endpunkt wurden zwei kombinierte Endpunkte gewählt: Zunahme der linksventrikulären Masse oder Tod und Verschlechterung der HRQOL oder Tod. Die intensivierte HD war mit einer Verbesserung in beiden kombinierten Endpunkten assoziiert: Zunahme der linksventrikulären Masse oder Tod (hazard ratio 0,61; 95\%-KI 0,46-0,82) und Verschlechterung der HRQOL oder Tod (hazard ratio 0,70; 95\% KI 0,53-0,92). Im Short Daily Trial wurden in der intensivierten HD-Gruppe die kombinierten Endpunkte durch einen Abnahme der linksventrikulären Masse und durch eine bessere HRQOL erreicht und nicht durch einen Rückgang der Mortalität (97).

Im Short Daily Trial konnten bei kurzer täglicher HD signifikant niedrigere prädialytische systolischen Blutdruckwerte trotz Reduktion der Antihypertensiva beobachtet werden. Die Serumphosphatwerte waren signifikant niedriger bei gleichzeitiger Reduktion der Phosphatbinder bei intensivierter HD $(97,101,102)$.

Die Patient*innen des Short Daily Trials wurden nach Studienende weiter beobachtet: Die Patient*innen, die in der intensivierten Gruppe waren, hatten im weiteren Verlauf eine geringere Mortalität bei einer medianen Nachbeobachtungszeit von 6,6 Jahren (103). 
Im Short Daily Trial zeigte sich eine signifikante Verbesserung der HRQOL. Das physische Gesundheitsempfinden verbesserte sich signifikant nach einem Jahr mit kurzer täglicher HD, während es in der konventionellen HD Gruppe konstant blieb $(97,104)$. Die Patient*innen mit kurzer täglicher HD erholten sich deutlich schneller nach den einzelnen Dialysesitzungen (initial 150 Minuten; nach 12 Monaten 60 Minuten) im Vergleich zu Patient*innen mit konventioneller HD (initial 120 Minuten; nach 12 Monaten 180 Minuten) (105).

Die Patient*innen mit intensivierter HD wiesen eine höhere Komplikationsrate hinsichtlich des AVShuntes auf. Mehr Interventionen wie Thrombektomien oder Shuntrevisionen waren mit kurzer täglicher HD notwendig (106).

\section{$\underline{\text { Nocturnal Trial }}$}

Im Nocturnal Trial der The Frequent Hemodialysis Trial Group wurden Patient*innen $(n=42)$, die mit konventioneller Heim-HD $(n=42)$ behandelt wurden, mit Patient*innen $(n=45)$, die mit täglich nächtlicher Heim-HD ( $n=45)$ behandelt wurden, verglichen (98). Beide Gruppen unterschieden sich nicht in den beiden Endpunkten Änderung der linkventrikulären Masse/Tod (hazard ratio 0,68; 95\%-KI 0,44-1,07) und Änderung der HRQOL/Tod (hazard ratio 0,91; 95\%-KI 0,58-1,43).

Im Nocturnal Trialkonnten signifikant niedrigere prädialytische systolische Blutdruckwerte bereits nach drei Monaten Studiendauer unter einer reduzierten Zahl an Antihypertensiva beobachtet werden (107). Die Serumphosphatspiegel war signifikant niedriger mit täglich nächtlicher HD; nur 27\% der Patient*innen benötigten noch Phosphatbinder am Studienende. Bei $42 \%$ der Patient*innen musste sogar Phosphat bei täglich nächtlicher HD substituiert werden (102).

Die renale Restfunktion ging mit täglich nächtlicher HD deutlich häufiger verloren als mit konventioneller HD. 67\% der Patient*innen mit täglich nächtlicher HD hatten nach einem Jahr keine Urinausscheidung mehr im Vergleich zu $36 \%$ der Patient*innen mit konventioneller HD (108).

Patient*innen der intensivierten Gruppe des Nocturnal Trial wiesen in der Nachbeobachtungszeit eine höhere Mortalität im Vergleich zur konventionellen HD auf (hazard ratio 3,88; 95\%-KI 1,2711,79) (109). In dieser Studie war die Mortalitätsrate in der Vergleichsgruppe mit konventioneller HD sehr niedrig mit 30 Todesfälle/1000 Patientenjahren. Die durchschnittliche Todesrate von Dialysepatient*innen ist meist 5-6-fach höher (110). Die Autoren vermuteten, dass dies zu einer Verzerrung der Ergebnisse führen könnte.

Die Rekonvaleszenz-Zeit nach der Dialysesitzung war kürzer mit täglich nächtlicher HD (initial 180 Minuten; nach 12 Monaten 30 Minuten) im Vergleich zur konventionellen HD (initial 180 Minuten; nach 12 Monaten 120 Minuten) (105). 


\section{$\underline{\text { Alberta Studie }}$}

In der Alberta Studie wurde der Rückgang der linksventrikulären Masse als Studienendpunkt gewählt. Nach sechs Monaten zeigte sich mit sechs Mal pro Woche nächtlicher HD ( $n=27)$ im Vergleich zur konventionellen HD $(n=25)$ ein signifikanter Unterschied in der linksventrikulären Masse (-15,3 g; 95\% KI 1,0- 29,6) (100). Die antihypertensive Medikation und die Menge an Phosphatbindern waren bei täglich nächtlicher HD geringer im Vergleich zur konventionellen HD. Die allgemeine HRQOL besserte sich nicht, aber spezifische Lebensbereiche (Belastung durch die Nierenerkrankung) zeigten eine Verbesserung mit intensivierter HD während der sechsmonatigen Studiendauer (100).

\section{$\underline{\text { ACTIVE-Studie }}$}

In der ACTIVE-Studie (A Clinical Trial of Intens/Ve Dialysis), wurden Patient*innen mit konventioneller HD (12h/Woche) ( $\mathrm{n}=98)$ mit Patient*innen mit intensivierter HD (24h/Woche) $(n=97)$ verglichen (99). Die HD wurde in beiden Studienarmen entweder im Dialysezentrum oder zu Hause über ein Jahr durchgeführt. Primärer Endpunkt der Studie war eine Veränderung der HRQOL. Die HRQOL war in beiden Studienarmen gleich, ebenso die Mortalität. Mit intensivierter HD waren weniger Antihypertensiva und Phosphatbinder notwendig. Die linksventrikuläre Masse war mit intensivierter HD signifikant niedriger im Vergleich zur konventionellen HD. Komplikationen am Gefäßzugang waren in beiden Gruppen gleich häufig (99).

\section{$\underline{\text { KDOQI-Leitlinie zu intensivierten HD-Verfahren }}$}

Die beiden Studien der The Frequent Hemodialysis Trial Group und die Alberta-Studie bilden die wissenschaftlich Grundlage, dass in der aktuellen KDOQI-Leitlinie die intensivierte HD neu als Therapieoption aufgenommen wurde (25):

- Patient*innen sollte die kurze tägliche Zentrums-basierte HD als Therapieoption angeboten werden im Hinblick auf eine potentielle Verbesserung der HRQOL und der körperlichen Aktivität.

- Patient*innen sollten über die Risiken der kurzen täglichen Zentrums-basierten HD aufgeklärt werden (Komplikationen am Gefäßzugang und potentielle intradialytische Hypotensionen).

- Wenn Patient*innen aus persönlichen Gründen täglich nächtliche Heim-HD präferieren, kann dies durchgeführt werden. Sie sollten über das Risiko der potentiell vermehrten Komplikationen am Gefäßzugang, der vermehrten Belastung des Umfeldes und dem möglicherweise schnelleren Verlust der renalen Restfunktion aufgeklärt werden.

- Schwangere Patientinnen sollten intensivierte HD erhalten. 


\section{$\underline{\text { Studien mit nächtlich intermittierender HD }}$}

Die nächtlich intermittierende HD, entweder im Dialysezentrum oder zu Hause durchgeführt, wurde bislang nicht prospektiv, randomisiert im Rahmen einer Studie mit konventioneller HD verglichen. Daher wird dieser Form der intensivierten HD auch keine Empfehlung von Seiten der KDOQI ausgesprochen, obwohl sie häufiger eingesetzt wird als die täglich nächtliche HD (25).

Ok et al. verglichen in einer prospektiven Fall-Kontroll-Studie Patient*innen mit Zentrumsbasierter nächtlich intermittierender HD $(n=247)$ mit Patient*innen mit Zentrums-basierter konventioneller HD ( $n=247)$ (111). Die Mortalitätsrate mit intermittierend nächtlicher HD war signifikant geringer im Vergleich zu konventioneller HD (1,77 versus 6,23 pro 100 Patientenjahre). Ok et al. beobachteten eine signifikante Reduktion des Serumphosphatwertes bei Patient*innen mit nächtlich intermittierender HD im Vergleich zu Patient*innen mit konventioneller HD. Bei Studienende nach 12 Monaten benötigten 83\% der Patient*innen mit konventioneller HD eine Medikation mit Phosphatbindern und nur 22\% der Patient*innen mit nächtlich intermittierender HD. Die linksventrikuläre Masse war bei den Patient*innen mit nächtlich intermittierender HD signifikant geringer im Vergleich zu Patient*innen mit konventioneller HD (111). Die HRQOL wurde bei Patient ${ }^{*}$ innen mit konventioneller HD in den Bereichen körperlicher Schmerz, mentale Gesundheit und Vitalität signifikant schlechter, während sie bei den Patient*innen mit nächtlich intermittierender HD konstant blieb (111).

Lacson et al. verglichen retrospektiv Patient*innen mit Zentrums-basierter nächtlich intermittierender HD ( $n=746)$ mit Patient*innen mit konventioneller HD ( $n=746)$ (112). Nach zwei Jahren war die Mortalitätsrate mit nächtlich intermittierender HD signifikant geringer als mit konventioneller Dialyse (27\% versus $19 \%$ ). Die Serumphosphatwerte waren signifikant niedriger mit nächtlich intermittierender HD im Vergleich zur konventionellen HD (112).

\subsubsection{Intensivierte Dialyseverfahren bei Kindern und Jugendlichen}

Es liegen keine prospektiven, randomisierten Studien zu intensivierten HD-Verfahren bei Kindern und Jugendlichen vor. Beobachtungsstudien stammen aus wenigen Zentren, die ihre Patient*innen vor und nach Umstellung auf intensivierte Verfahren analysierten (113-118) (Tabelle 2).

Die kurze tägliche HD wurde in Straßburg (Frankreich) von Fischbach et al. als Zentrums-basierte tägliche online HDF initiiert (113). Mit kurzer täglicher online HDF konnte nach 12 Monaten ein signifikanter Abfall des Serumphosphats von 1,9 auf 1,3 mg/dl trotz Reduktion der Phosphatbinder und Aufhebung diätetischer Restriktionen beobachtet werden. Ebenfalls sank der mittlere arterielle Blutdruck signifikant nach Umstellung von konventioneller HDF zu kurzer täglicher HDF (von $95 \mathrm{mmHg}$ auf $87 \mathrm{mmHg}$ ) und die interventrikuläre kardiale Septumdicke verringerte sich signifikant (von 10,9 auf 6,8 mm) (113). Die mittlere Wachstumsgeschwindigkeit nahm nach Umstellung auf kurze tägliche online HDF von $3,8 \mathrm{~cm} / \mathrm{Jahr}$ auf $14,3 \mathrm{~cm} / \mathrm{Jahr}$ innerhalb 
eines Jahres zu (114). Camargo et al. konnten diese positiven Effekte auf das Wachstum ebenfalls beobachten (115).

Geary et al. etablierten ein täglich nächtliches Heim-HD Programm für Kinder und Jugendliche (119). Vier jugendliche Dialysepatient*innen wurden in die erste Analyse eingeschlossen (117). Sämtliche diätetische und Flüssigkeitsrestriktionen konnten nach Umstellung von konventioneller HD aufgehoben werden. Aufgrund von niedrigen Serumphosphat- und Serumcalciumwerten mussten Calcium und Phosphat substituiert werden. Die arteriellen systolischen Blutdruckwerte waren bei den vier Patient*innen nach Umstellung auf täglich nächtliche Heim-HD sehr unterschiedlich: Zwei Patient*innen benötigten weiterhin Antihypertensiva; die anderen beiden hatten häufig hypotensive Episoden an der Dialyse (117). Die HRQOL verbesserte sich bei drei der vier Patient*innen. Die Schulfehltage wurden nach Umstellung auf täglich nächtliche HeimHD weniger. Allerdings war die Belastung für die Familien mit der täglichen Heim-HD erheblich (117).

Hothi et al. untersuchten vier Jugendliche mit täglich nächtlicher HD. Bei allen vier Patient*innen konnten Phosphatbinder abgesetzt werden. Die Serumphosphatwerte lagen im Normbereich bei normaler Kost ohne Restriktionen. Die Parathormonspiegel lagen bei drei der vier Patient ${ }^{\star}$ innen mit täglich nächtlicher HD unter 100 pg/ml (118).

\begin{tabular}{|c|c|c|c|c|c|c|c|c|}
\hline HD & Studie & $\begin{array}{l}\text { Blut- } \\
\text { druck }\end{array}$ & $\begin{array}{c}\text { Serum- } \\
\text { phosphat }\end{array}$ & $\begin{array}{l}\text { Lebens- } \\
\text { qualität }\end{array}$ & $\begin{array}{l}\text { Wach- } \\
\text { stum }\end{array}$ & BMI & Diät & $\begin{array}{l}\text { Schul- } \\
\text { fehltage }\end{array}$ \\
\hline \multirow[t]{3}{*}{$\begin{array}{l}\text { Kurze } \\
\text { tägliche }\end{array}$} & $\begin{array}{l}\text { Fischbach } \\
\text { et al. (113) }\end{array}$ & $\downarrow$ & $\downarrow$ & $\uparrow$ & & & keine & $\downarrow$ \\
\hline & $\begin{array}{l}\text { Fischbach } \\
\text { et al. (114) }\end{array}$ & & & & $\uparrow$ & $\uparrow$ & & \\
\hline & $\begin{array}{l}\text { Camargo } \\
\text { et al. (115) }\end{array}$ & & & & $\uparrow$ & & & \\
\hline \multirow[t]{2}{*}{$\begin{array}{l}\text { Täglich } \\
\text { nächtliche }\end{array}$} & $\begin{array}{l}\text { Geary et al. } \\
\text { (117) }\end{array}$ & $\sim \downarrow$ & $\downarrow$ & $\uparrow$ & $\sim$ & $\uparrow$ & keine & $\downarrow$ \\
\hline & $\begin{array}{l}\text { Hothi et al. } \\
(118)\end{array}$ & & $\downarrow$ & & & & keine & \\
\hline
\end{tabular}

Tabelle 2 Intensivierte HD-Verfahren bei Kinder und Jugendlichen 


\subsection{Fragestellung}

Bei erwachsenen Patient*innen stellen intensivierte HD-Verfahren eine Option dar, die Morbidität und Lebensqualität der Betroffenen zu verbessern. Um zu untersuchen, ob diese positiven Effekte auch bei Kindern und Jugendlichen zu beobachten sind, wurde ein nächtliches intermittierendes HD-Programm als prospektive Beobachtungsstudie initiiert. Es wurde untersucht, welchen Einfluss die nächtlich intermittierende HD auf Urämie-assoziierte Parameter, auf die Medikamentenmenge und auf die Lebensqualität der Betroffenen im Vergleich zur konventionellen HD und zur PD hat. Des Weiteren wurde versucht, ob die nächtliche intermittierende HDF gegenüber der nächtlich intermittierenden HD die Dialysequalität weiter verbessert.

Um zu analysieren, in wieweit intensivierte HD-Verfahren bereits im klinischen Alltag sowohl bei Kindern und Jugendlichen als auch bei Erwachsenen eingesetzt werden, und welche Einstellung die Behandler gegenüber diesen Verfahren haben, wurden Umfragen unter Erwachsenennephrolog*innen und Kindernephrolog*innen durchgeführt. 


\section{Ergebnisse}

2.1. Aufbau eines Zentrums-basierten, nächtlich intermittierenden Hämodialyseprogramms für Kinder und Jugendliche („A hospital-based intermittent nocturnal hemodialysis program for children and adolescents“)

Der nachfolgende Text entspricht inhaltlich dem Abstract der Arbeit (120) (J Pediatr 2011; 158(1):95-96; https://doi.org/10.1016/i.jpeds.2010.06.036):

„ZIEL: Etablierung eines Zentrums-basierten nächtlichen HD-Programms für Kinder und Jugendliche.

STUDIENDESIGN: Sechzehn Patient*innen (Alter 0,5 bis 17 Jahre) wurden prospektiv eingeschlossen. Urämie-assoziierte Laborwerte, Anzahl und Dosierung von Medikamenten sowie die HRQOL wurden analysiert.

ERGEBNISSE: Die nächtlich intermittierende HD wurde gut vertragen. Kt/V und nPNA (normalized protein nitrogen appearance) waren signifikant höher bei nächtlich intermittierender HD. Der prädialytische mittlere arterielle Druck, die Harnstoff-, Phosphat- und Parathormonspiegel waren deutlich niedriger. Die diätetischen und die Flüssigkeitsrestriktionen konnten bei nächtlich intermittierender HD aufgehoben werden. Die Anzahl und Dosierung von Antihypertensiva, Phosphat- und Kaliumbindern konnten reduziert werden. Die HRQOL verbesserte sich und die Schulfehltage wurden bei allen Patient*innen weniger.

SCHLUSSFOLGERUNGEN: Zusätzlich zu einer besseren Kontrolle von Urämie-assoziierten Symptomen ermöglicht die nächtlich intermittierende HD eine Nahrungs- und Flüssigkeitsaufnahme ohne Einschränkungen. Das Wohlbefinden der Patient*innen verbessert sich. Angesichts des anhaltenden Mangels an Spenderorganen und der hohen Morbidität und Mortalität bei konventioneller HD sind intensivierte Dialysemodalitäten eine dringend benötigte Therapieoption." (Übersetzung der Autorin) 


\subsection{Hämodiafiltration in einem Zentrum-basierten nächtlich intermittierenden Dialyseprogramm für Kinder und Jugendliche (,Hemodiafiltration in a pediatric nocturnal dialysis program”)}

Nach Etablierung des nächtlich intermittierenden HD-Programms für Kinder und Jugendliche wurde in einer prospektiven Studie untersucht, ob HDF im Vergleich zur HD die Qualität der Dialyse noch weiter steigern kann.

Der nachfolgende Text entspricht inhaltlich dem Abstract der Arbeit (121) (Pediatr Nephrol 2014; 29(8):1411-1416; https://doi.org/10.1007/s00467-014-2776-9):

„HINTERGRUND: Um die nachteiligen Folgen der konventionellen Dialyse zu überwinden, wurden intensivierte Dialyseprogramme entwickelt. Deren Machbarkeit und positive Auswirkungen im pädiatrischen Bereich wurde bereits gezeigt. Um zu untersuchen, ob ein solches intensiviertes HDProgramm weiter verbessert werden kann, wurde die HDF in ein Zentrum-basiertes nächtliches HDProgramm für Kinder und Jugendliche implementiert.

METHODEN: Nach Beginn der konventionellen HD wechselten sieben Patient*innen für drei Monate zur intermittierend nächtlichen HD, weitere drei Monate zur intermittierend nächtlichen online HDF und schließlich zurück zur nächtlichen HD. Urämie assoziierte Parameter, prädialytischer Blutdruck, intradialytische Ereignisse, Albumin, Vitamine und Spurenelemente wurden untersucht. Kt/V und nPNA wurden analysiert. Die Menge an Phosphatbindern, Kaliumbindern und Antihypertensiva wurden registriert. Diätetische Einschränkungen wurden ebenfalls dokumentiert.

ERGEBNISSE: Die Phosphatwerte und Parathormonspiegel waren nach dem Wechsel von konventioneller HD zur nächtlichen HD und zur nächtlichen HDF signifikant reduziert. Die Dialysedosis $(\mathrm{Kt} / \mathrm{V})$ war bei den Patient*innen unter nächtlicher HD und nächtlicher HDF signifikant erhöht. Mit nächtlicher HDF erhöhte sich das Kt/V auch signifikant im Vergleich zur nächtlichen HD. Der Blutdruck war bei den Patient*innen sowohl mit nächtlicher HD als auch mit nächtlicher HDF trotz Reduktion der blutdrucksenkenden Medikamente signifikant niedriger. Serumalbuminspiegel und nPNA waren signifikant höher mit nächtlicher HD und mit nächtlicher HDF. Vitamine und Spurenelemente blieben unverändert. Alle diätetischen Einschränkungen konnten bei den Patient*innen sowohl mit nächtlicher HD als auch mit nächtlicher HDF aufgehoben werden.

SCHLUSSFOLGERUNGEN: Die Einführung eines nächtlichen HD-Programms verbesserte die Urämieassoziierten Parameter, die Ernährung und die hämodynamische Stabilität der Patient*innen signifikant. Die HDF konnte das intensivierte Programm durch Erhöhung des Kt/V noch weiter verbessern." (Übersetzung der Autorin) 


\subsection{Vergleich der nächtlichen Peritonealdialyse mit nächtlich intermittierender Hämodialyse bei}

Kinder und Jugendlichen (,,Is peritoneal dialysis still an equal option? Results of the Berlin pediatric nocturnal dialysis program")

Die nächtlich intermittierende HD ist vor allem für ältere Kinder und Jugendliche geeignet. Eine weitere nächtliche Dialyseoption ist die nächtlich automatisierte PD. In dieser Studie wurde verglichen, ob die nächtlich intermittierende HD der PD bei Jugendlichen überlegen ist.

Der nachfolgende Text entspricht inhaltlich dem Abstract der Arbeit (122) (Pediatr Nephrol 2015; 30(7):1181-1189; https://doi.org/10.1007/s00467-015-3043-4):

„HINTERGRUND: Die PD und die konventionelle HD gelten als gleichermaßen effiziente Dialyseverfahren bei Kindern und Jugendlichen. Das Ziel unserer Studie war zu analysieren, ob ein intensiviertes, nächtliches HD-Programm der PD bei jugendlichen Dialysepatient*innen überlegen ist.

METHODEN: 13 Patient*innen wurden prospektiv in das intensivierte, nächtliche HD-Programm aufgenommen. Urämie-assoziierte Parameter, Parameter für Ernährung, Medikamente und Blutdruck wurden analysiert. Diese Daten wurden mit 13 PD-Patient*innen gepaart nach Geschlecht, Alter und Gewicht, zu Beginn des jeweiligen Dialyseprogramms und nach sechs Monaten Dialyse verglichen.

ERGEBNISSE: Die Serumphosphatspiegel nahmen in der nächtlichen HD-Gruppe signifikant ab und blieben in der PD-Gruppe unverändert während der 6-monatigen Beobachtungsphase. Der arterielle Blutdruck war in der nächtlichen HD-Gruppe signifikant niedriger trotz Reduktion der Antihypertensiva, während die Blutdruckwerte in der PD-Gruppe unverändert blieben. Eine präexistierende linksventrikuläre Hypertrophie war nach sechs Monaten nächtlicher HD nicht mehr nachweisbar; die Serumalbuminwerte verbesserten sich. Bei den Patient*innen mit nächtlicher HD konnten diätetische Einschränkungen aufgehoben werden, während sie für die Patient*innen mit PD-Behandlung bestehen blieben. Die Restdiurese blieb nach sechs Monaten sowohl mit nächtlicher HD als auch mit PD unverändert. Patient*innen mit nächtlicher HD waren signifikant weniger Tage im Krankenhaus im Vergleich zu den PD-Patient*innen.

SCHLUSSFOLGERUNGEN: Basierend auf diesen Ergebnissen führt die nächtliche, intensivierte HD zu signifikant verbesserten Urämie assoziierten Parametern. Wenn individuell und logistisch möglich, sollte die nächtliche intensivierte HD bei älteren Kindern und Jugendlichen bevorzugt eingesetzt werden." (Übersetzung der Autorin) 


\subsection{Implementierung und Hindernisse von intensivierten Hämodialyseverfahren bei Kindern und}

Jugendlichen weltweit („,Barriers for implementation of intensified hemodialysis: survey results from the International Pediatric Dialysis Network")

Berichte über den Einsatz von intensivierten HD-Verfahren bei Kindern und Jugendlichen gibt es nur aus einzelnen pädiatrischen Dialysezentren. Mittels einer Umfrage innerhalb des International Pediatric Dialysis Network wurde in dieser Arbeit analysiert, in wieweit intensivierte HD-Verfahren im klinischen Altag weltweit bereits eingesetzt werden.

Der nachfolgende Text entspricht inhaltlich dem Abstract der Arbeit (123) (Pediatr Nephrol 2018; 33(4):705-712;https://doi.org/10.1007/s00467-017-3831-0):

„HINTERGRUND: Bei Patient*innen unter konventioneller HD ist die Morbidität hoch und die Lebensqualität stark beeinträchtigt. Intensivierte HD-Programme wurden entwickelt, um diese Unzulänglichkeiten zu überwinden. Bislang haben nur wenige pädiatrische Dialysezentren über die Implementierung eines solchen intensivierten HD-Programms berichtet.

METHODEN: Eine Online-Umfrage wurde an alle 221 pädiatrischen Dialysezentren gesendet, die am International Pediatric Dialysis Network teilnehmen. Ziel der Untersuchung war es, die Einstellung von pädiatrischen Nephrolog*innen zu intensivierten HD-Verfahren, die Häufigkeit des Einsatzes im klinischen Alltag und die Hürden für den Einsatz zu analysieren.

ERGEBNISSE: Von 221 pädiatrischen Dialysezentren antworteten $61 \%(n=134)$ auf die Umfrage. 69\% gaben an, dass sie genügend Evidenz für den Einsatz von intensivierten HD-Verfahren sehen, unabhängig davon, ob intensivierte Verfahren in ihrem eigenen Zentrum bereits angeboten wurden oder nicht. 50\% der Befragten hielten die täglich nächtliche HD für das beste HD-Verfahren. Nur $2 \%$ der Befragten hielten die konventionelle HD für das beste Verfahren. Insgesamt gaben $38 \%$ der Befragten an, dass in ihrem Zentrum nur einzelne Patient*innen mit intensivierter HD behandelt werden, am häufigsten in Form von kurzen täglichen HD-Sitzungen. Die wichtigsten Hindernisse für den Ausbau intensivierter HD-Programme waren der Mangel an ausreichenden Finanzmitteln (66\%) und der Mangel an Personal (63\%).

FAZIT: Die intensivierte HD wird von vielen pädiatrischen Nephrolog*innen als die Dialysemodalität, die mit dem besten Outcome assoziiert ist, angesehen. Die eingeschränkte Anwendung intensivierter HDVerfahren zeigt, wie wichtig es ist, die Hindernisse, die dem Einsatz im klinischen Alltag im Wege stehen, zu beseitigen." (Übersetzung der Autorin) 


\subsection{Implementierung und Hindernisse von intensivierten Hämodialyseverfahren in Deutschland}

(„Attitudes of nephrologists towards intensified hemodialysis“)

Bei erwachsenen Dialysepatient*innen gibt es mehrere pro- und retrospektive Studien, die den Einsatz von intensivierten HD-Verfahren untersuchten. Allerdings gibt es keine Angaben, inwieweit intensivierte HD im klinischen Alltag etabliert ist und welche Hindernisse dem Einsatz im Wege stehen. Um die Situation in Deutschland zu evaluieren, wurden in folgender Arbeit die Mitglieder der DGfN befragt.

Der nachfolgende Text entspricht inhaltlich dem Abstract der Arbeit (124) (Clin Nephrol 2018; 90(4):255261; https://doi.org/10.5414/CN109350)

„ZIELE: Intensivierte HD-Programme wurden entwickelt, um die Mängel der konventionellen HD zu kompensieren. Es liegen jedoch keine Daten zur Implementierung von intensivierten HD-Programmen im klinischen Alltag vor. Deshalb wurde die Einstellung von Nephrolog*innen gegenüber intensivierten HD-Verfahren, die Häufigkeit des Einsatzes der intensivierten Verfahren im klinischen Alltag und die Hürden für die Umsetzung erfragt.

MATERIAL UND METHODEN: Es wurde eine Online-Umfrage innerhalb der DGfN durchgeführt.

ERGEBNISSE: Unter den Befragten gaben 44\% an, dass sie genügend Evidenz für den Einsatz von intensivierten HD-Verfahren sahen, unabhängig davon, ob intensivierte Verfahren in ihrem eigenen Zentrum bereits angeboten wurden oder nicht. Die wichtigsten Hindernisse für den Ausbau intensivierter HD-Programme waren eine unzureichende Finanzierung (81\%) und der Mangel an Personal (60\%). $44 \%$ der Befragten boten intensivierte HD-Verfahren bereits an. Es erhielten vor allem Patient*innen eine intensivierte Dialyse, wenn sie unter konventioneller HD unzureichend dialysiert waren. Nur 19\% der Befragten boten routinemäßig allen Patient*innen in Vorbereitung auf eine NET ein intensiviertes Verfahren an. 33\% der Befragten boten kurze tägliche, $70 \%$ nächtlich intermittierende und $4 \%$ täglich nächtliche HD an. In 30\% der befragten Zentren wurde die intensivierte HD zu Hause durchgeführt.

FAZIT: Fast die Hälfte der Befragten bot intensivierte HD-Verfahren an. Die inadäquate Finanzierung von intensivierten HD-Programmen repräsentierte die wichtigste Barriere für den breiten Einsatz im klinischen Alltag." (Übersetzung der Autorin) 


\subsection{Implementierung von assistierten Dialyseverfahren in Deutschland („,Attitudes of nephrologists towards assisted home dialysis in Germany")}

Häusliche Dialyseverfahren stellen sowohl für Patient*innen als auch aus ökonomischer Sicht eine gute Option im Vergleich zur Zentrums-basierten konventionellen HD dar. Die Heimdialyse kann für die Patient*innen und die Angehörigen eine starke Belastung bedeuten. Eine Assistenz durch ambulante Pflegedienste, Pflegekräfte einer Wohneinrichtung oder des Dialysezentrums kann für die Betroffenen eine Erleichterung darstellen. Inwieweit assistierte Heim-Dialyseverfahren in Deutschland etabliert sind, wurde in folgender Arbeit untersucht.

Der nachfolgende Text entspricht inhaltlich dem Abstract der Arbeit (125) (Clin Kidney J 2018; 11(3):400-405; https://doi.org/10.1093/ckj/sfx108)

„HINTERGRUND: Die assistierte Heimdialyse ist eine Option, die Vorteile der Heimdialyse-Therapie auch den Dialysepatient*innen zu ermöglichen, die allein nicht in der Lage sind, eine Heimdialyse durchzuführen. Die assistierte Heimdialyse wird in vielen Ländern zunehmend angeboten. Aus Deutschland gibt es hierzu keine Daten.

METHODEN: Eine Online-Umfrage wurde durchgeführt, um die Einstellungen und Hindernisse für die Umsetzung von assistierten Dialyseverfahren zu analysieren. Die Umfrage wurde an alle 2060 Mitglieder der DGfN versandt.

ERGEBNISSE: $14 \%$ der Befragten $(n=286)$ antworteten. Dies entsprach $24 \%$ aller deutschen Dialysezentren. Assistierte Heimdialyse wurde als eine sinnvolle Option von über $90 \%$ der Antwortenden angesehen. 55\% der Zentren boten assistierte Heimdialyse-Verfahren an (bevorzugt PD). Die Anzahl der Patient*innen, die mit einem assistierten Heimdialyse-Verfahren behandelt wurden, war gering. $77 \%$ der Zentren behandelten nicht mehr als 10 Patient*innen damit. Nephrol*innen, die assistierte Heimdialyse-Verfahren anboten, waren älter und arbeiteten in größeren Dialysezentren im Vergleich zu Nephrolog*innen, die diese Verfahren nicht anboten. Assistierte Dialyseverfahren wurden in 57\% der Zentren im Prädialysestadium angeboten. Unzureichende konventionelle HD und Patientenwunsch waren Gründe für das Angebot der assistierten Verfahren. Hindernisse für das Angebot assistierter Heimdialyse stellten mangelnde Kostenerstattung, Personalmangel und mangelnde Fachkompetenz dar.

SCHLUSSFOLGERUNGEN: Aus Sicht deutscher Nephrolog*innen sind assistierte Dialyseverfahren eine sinnvolle Methode für die häusliche Dialyseversorgung. Unzureichende Finanzierung und Mangel an qualifiziertem Personal wurden als wichtigste Hindernisse für den Einsatz assistierter Verfahren gesehen. Weitere Studien sind notwendig, um assistierte Heimdialyse-Verfahren im Hinblick auf Patienten Outcome und Kosten zu evaluieren." (Übersetzung der Autorin) 


\section{Diskussion}

\subsection{Kardiovaskuläre Surrogat-Parameter bei intensivierten HD-Verfahren}

Kardiovaskuläre Komplikationen sind der Hauptgrund für die deutlich erhöhte Mortalität von Dialysepatient*innen im Vergleich zur Allgemeinbevölkerung. Dies trifft für alle Altersgruppen vom Säugling bis hin zum hochbetagten Erwachsenen zu (110). Da kardiovaskuläre Ursachen auch nach Transplantation eine der Haupttodesursachen darstellen und die urämische Vaskulopathie nach Nierentransplantation nicht komplett reversibel ist, sollten alle Anstrengungen ab dem frühen Stadium der CNI und während der Dialysezeit unternommen werden, um vaskuläre Umbauprozesse zu verhindern.

Bei über $60 \%$ der Kinder und Jugendlichen lassen sich bei Dialysebeginn hypertensive arterielle Blutdruckwerte feststellen (126). Auch Dyslipidämien treten bei Kindern und Jugendlichen mit CNI im Vergleich zur gleichaltrigen Normalbevölkerung signifikant häufiger auf (127). Neben diesen klassischen Risikofaktoren, arterielle Hypertonie und Dyslipidämie, trägt in der CNI die Imbalance des Calcium-Phosphat-Stoffwechsels wesentlich zur Pathogenese kardiovaskulärer Erkrankungen bei (128).

Als kardiovaskuläre Surrogat-Parameter werden in Studien häufig der arterielle Blutdruck, die linksventrikuläre Masse, Anzahl der Antihypertensiva, sowie funktionelle und strukturelle Gefäßveränderungen untersucht. Als Maß für den Calcium-Phosphat-Stoffwechsel werden der Serumphosphatspiegel, der Parathormonspiegel und die Menge an Phosphatbindern analysiert.

Im Berliner Nachtdialyseprogramm für Kinder und Jugendliche zeigten sich signifikant positive Effekte auf kardiovaskuläre Surrogat-Parameter (120-122). Nach Umstellung von konventioneller HD auf nächtlich intermittierende HD sank der mediane Serumphosphatwert von 2,1 auf 1,4 mmol/l trotz Reduktion der Phosphatbinder und Aufhebung der diätetischen Phosphatrestriktion bei 16 Patient*innen (120). Der mediane Parathormonspiegel sank von 445 auf $184 \mathrm{ng} / \mathrm{l}$. Der prädialytische mittlere arterielle Blutdruck verringerte sich von 102 auf $93 \mathrm{mmHg}$ trotz Reduktion der antihypertensiven Medikation. Bei Einschluss in das Nachtdialyseprogramm wiesen zwei Patienten eine linksventrikuläre Hypertrophie auf, die nach sechs Monaten nächtlich intermittierender HD nicht mehr nachweisbar war (120).

In einer zweiten Studie innerhalb des Berliner Nachtdialyseprogrammes wurden sieben Patient*innen prospektiv in einem cross-over Design mit konventioneller HD, mit nächtlich intermittierender HD und mit nächtlich intermittierender online HDF untersucht (121). Die Serumphosphatspiegel sanken auch hier signifikant sowohl bei nächtlich intermittierender HD ($0,4 \mathrm{mmol} / \mathrm{l})$ als auch bei nächtlich intermittierender online HDF $(-0,5 \mathrm{mmol} / \mathrm{l})$ im Vergleich zur konventionellen $\mathrm{HD}$ ab trotz Absetzen der Phosphatbinder und Aufhebung diätetischer Restriktionen. Der prädialytische mittlere arterielle Blutdruck sank signifikant um $8 \mathrm{mmHg}$ nach Umstellung auf nächtlich intermittierende HD mit reduzierter antihypertensiver Therapie (121). 
In der dritten Studie wurden Jugendliche mit nächtlich intermittierender HD mit Jugendlichen mit nächtlich automatisierter PD retrospektiv verglichen (122). Es wurden die Unterschiede in beiden Gruppen vor Beginn der Dialyse und nach sechs Monaten Dialyse analysiert. Bei nächtlich intermittierender HD sank der prädialytische mittlere arterielle Blutdruck trotz Reduktion der antihypertensiven Medikation signifikant um $6 \mathrm{mmHg}$ ab, während bei PD der mittlere arterielle Blutdruck konstant blieb. Bei drei Patienten mit zuvor beschriebener linksventrikulärer Hypertrophie normalisierte sich die Wanddicke nach Beginn der nächtlich intermittierenden HD. Der Serumphosphatspiegel war nach sechs Monaten nächtlich intermittierender HD signifikant niedriger (-0,8 mml/l). Unter PD änderte er sich nicht (122).

Diese Ergebnisse bestätigten die positiven Auswirkungen wie sie bereits für Erwachsene mit nächtlich intermittierender HD gezeigt werden konnten. Ok et al. beschrieben eine signifikante Reduktion des Serumphosphates bei zeitgleicher Reduktion der Phosphatbinder bei Patient*innen mit nächtlich intermittierender HD im Vergleich zu Patient*innen mit konventioneller HD. Die linksventrikuläre Masse war mit nächtlich, intermittierender HD signifikant geringer (111). Lacson et al. berichteten über signifikant niedrigere Serumphosphatspiegel mit nächtlich intermittierender HD im Vergleich zur konventionellen HD (112). Weitere Beobachtungsstudien mit kleinen Fallzahlen berichteten ebenfalls positive Effekte auf arteriellen Blutdruck und Phosphatwerte (129-133). Nach einem Jahr nächtlich intermittierender HD konnte bei Erwachsenen gezeigt werden, dass sich die flussvermittelte Vasodilatation, als Maß für eine bessere Endothelfunktion, im Vergleich zur konventionellen HD signifikant steigerte (134). Eine höhere flussvermittelte Vasodilatation war mit niedrigeren Phosphatserumwerten assoziiert (134).

Auch mit anderen Modalitäten der intensivierten HD wie der kurzen täglichen HD und der täglich nächtlichen HD konnten positive Effekte auf kardiovaskuläre Surrogat-Parameter beobachtet werden $(97,98,101,102,107,113,117,118,135-138)$. Strukturelle Gefäßveränderungen wurden von Yuen et al. analysiert: 38 Patient*innen wurden prospektiv mit täglich nächtlicher Heim-HD auf Progression in einer Computertomografie nachgewiesenen koronaren Verkalkungen untersucht (139). Innerhalb eines Jahres kam es bei Patient*innen mit präexistierenden ausgeprägten Verkalkungen nur zu einer minimalen, nicht signifikanten Progression der Verkalkungen. Die Autoren interpretierten die nicht signifikante Progression als positiven Effekt der intensivierten HD (139).

Die positiven Effekte auf den arteriellen Blutdruck und die Calcium-Phosphat-Homöostase, die bei Kindern und Jugendlichen mit intermittierend nächtlicher HD erzielt werden konnten, sprechen für eine deutliche Verbesserung des kardiovaskulären Risikoprofils. Funktionelle und strukturelle Gefäßveränderungen wurden in diesen drei Studien nicht untersucht. Aufgrund der Ergebnisse bei erwachsenen Patient*innen mit intensivierten Dialyseverfahren $(134,139)$ ist es sehr wahrscheinlich, dass sich bei Kindern und Jugendlichen mit Hilfe intensivierter HD-Verfahren 
vaskuläre Umbauprozesse ebenfalls verzögern lassen. Dies legt nahe, dass sich die Langzeitprognose der Patient*innen mit intensivierter HD erheblich verbessern kann.

\subsection{Lebensqualität bei intensivierten HD-Verfahren}

Die Dialyse als medizinische Therapie stellt für die Betroffenen und ihrem direkten sozialen Umfeld häufig eine erhebliche Belastung dar. Nachdem das primäre Überleben der Betroffenen aufgrund der besseren Dialysetechnik in den letzten Jahren nicht mehr allein primäres Behandlungsziel ist, rückt die Lebensqualität der Patient*innen zunehmend ins Blickfeld der medizinischen Forschung. Gemäß der Weltgesundheitsorganisation ist Lebensqualität die subjektive Wahrnehmung einer Person über ihre Stellung im Leben, in Relation zur Kultur und den Wertesystemen, in denen sie lebt, in Bezug auf ihre Ziele, Erwartungen und Standards (140). Unterschieden wird zwischen einer allgemeinen Lebensqualität und der HRQOL. Letztere ist definiert als der subjektiv wahrgenommene Gesundheitszustand des Betroffenen. Bei der Bewertung spielt die Fähigkeit den Alltag zu bewältigen und sich sozial zu integrieren die wichtigste Rolle (141). Die HRQOL ist variabel im zeitlichen Verlauf. Sie ist abhängig vom Krankheitsverlauf, der durchgeführten medizinischen Therapien und dem mentalen Anpassungsprozess des Individuums (141). Die gezielte Untersuchung der HRQOL ist auch deshalb von Interesse, da chronisch Kranke nicht automatisch in ihrer HRQOL beeinträchtigt sind. Viele Patient*innen mit chronischen Erkrankungen berichten über ein mit gesunden Probanden vergleichbare HRQOL (142). Als Messinstrumente wurden Fragebögen entwickelt, die entweder krankheitsübergreifend oder krankheitsspezifisch die HRQOL ermitteln. Die Fragebögen werden je nach Verfahren selbständig schriftlich ausgefüllt oder im Interviewverfahren vervollständigt. Von den krankheitsübergreifenden Verfahren wurde in Studien zu intensivierten HD-Verfahren häufig der SF-36 eingesetzt (143). Diesen Test gibt es sowohl in der Version zur Selbst- als auch zur Fremdbeurteilung. Er lässt sich bei Personen ab 14 Jahren einsetzen. Für Dialysepatient*innen gibt es als krankheitsspezifische Testverfahren den Kidney Disease Quality of Life (KDQOL) Test (144).

Die HRQOL spielt für Dialysepatient*innen eine wichtige Rolle. In einer Umfrage innerhalb des Kuratoriums für Dialyse und Nierentransplantation KfH wurden 4518 HD-Patient*innen befragt, welche Aspekte innen bei der Dialysebehandlung wichtig sind. Über $90 \%$ der Befragten empfanden die Sicherheit der Behandlung, die HRQOL und die Zufriedenheit mit der Versorgung in der Dialyseeinrichtung als sehr wichtig. Die Lebenserwartung, die Fahrtzeiten zur Klinik und die Vereinbarkeit mit dem Beruf wurden als weniger wichtig eingestuft (145). Urquart-Secord et al. befragten erwachsene HD-Patient*innen und die Angehörigen, die die Patient*innen im häuslichen Umfeld versorgten, anhand welcher Kriterien sie den Erfolg der Dialysebehandlung messen. Kontrolle der Müdigkeit, Krankheitsbewältigung, Möglichkeit zu Reisen und Dialyse-freie Zeit waren die wichtigsten Faktoren (146). 
Im Berliner Nachtdialyseprogramm zeigte sich eine deutlich verbesserte HRQOL. Bei Etablierung des Programmes wurden die HRQOL der Patient*innen vor Umstellung und nach vier Monaten mit nächtlich intermittierender HD mittels SF-36 ermittelt. Die HRQOL besserte sich deutlich sowohl in der Selbsteinschätzung als auch in der Einschätzung der Eltern und des oder der betreuenden Kindernephrolog*in. Zuvor an konventioneller HD verordnete diätetische und Flüssigkeitsrestriktionen konnten aufgehoben werden. Zudem verringerten sich die Schulfehltage von 37 auf 12 innerhalb von sechs Monaten (120). In den beiden weiteren Studien wurde die HRQOL nicht systematisch mittels standardisiertem Testverfahren untersucht. Auch in diesen Studien konnten alle Flüssigkeits- und diätetischen Restriktionen aufgehoben werden, was für eine verbesserte HRQOL gemäß des KDQOL-Testverfahrens spricht (144). Die Tage, die die Patient*innen stationär im Krankenhaus waren, waren mit nächtlich intermittierender HD deutlich geringer als bei Gleichaltrigen mit nächtlicher PD (122).

Die Verlagerung der Dialysezeit in die Nacht ermöglicht es den Jugendlichen nicht nur die komplette Unterrichtszeit in der Schule anwesend zu sein, sondern auch am Nachmittag ihren Freizeitaktivitäten nachzugehen. Die nächtlichen Verfahren stellen für Jugendliche und junge Erwachsene eine gute Option dar, um möglichst uneingeschränkt am Ausbildungs- bzw. Berufsalltag teilhaben zu können. Mit konventioneller HD können viele Patient*innen ihrem Beruf nicht mehr nachgehen. In einer US-amerikanischen Studie arbeiteten nach einem Jahr konventioneller HD nur noch ein Drittel der Dialysepatient*innen, die vor Beginn der Dialyse berufstätig gewesen waren (147).

Andere Zentren, die intensivierte HD-Verfahren bei Kindern und Jugendlichen einsetzten, berichteten ebenfalls über eine deutlich verbesserte HRQOL nach Umstellung auf ein intensiviertes Verfahren (113,117). Fischbach et al. analysierte die HRQOL für die kurze tägliche HDF nicht systematisch mittels eines standardisierten Testverfahrens (113). Er berichtete über eine Steigerung der körperlichen Aktivität und eine geringere Fehlzeit in der Schule. Bei allen Patient*innen konnten diätetische und Flüssigkeitsrestriktionen mit kurzer täglicher HDF aufgehoben werden (113). Mit täglich nächtlicher HD besserte sich die HRQOL bei Kindern und Jugendlichen ebenfalls (117). Geary et al. konnten bei drei der vier Patient*innen eine verbesserte HRQOL ermitteln. Die Schulfehltage der Patient*innen wurden weniger und die Lehrer der Patient*innen berichteten im Interview sowohl über eine bessere Teilhabe am Unterricht als auch über vermehrte soziale Kontakte innerhalb der Schule. Die aufgehobenen Flüssigkeits- und diätetischen Restriktionen trugen zusätzlich zur Verbesserung der HRQOL bei (117).

Welchen Einfluss intensivierte HD-Verfahren auf die HRQOL haben, wurde in mehreren Studien bei Erwachsenen ebenfalls untersucht. Im Short Daily Trial zeigte sich eine signifikante Verbesserung der HRQOL und kürzere Rekonvaleszenz-Zeiten nach den Dialysesitzungen $(97,104,105)$. Im Nocturnal Trial zeigte sich kein signifikanter Einfluss auf die HRQOL im Vergleich der beiden Gruppen konventionelle HD und täglich nächtliche HD (98). Die Rekonvaleszenz-Zeit nach der Dialysesitzung war kürzer mit täglich nächtlicher HD im Vergleich zur konventionellen HD (105). 
In einer multizentrischen spanischen Studie konnte gezeigt werden, dass die HRQOL bei Patient*innen mit kurzer täglicher HD signifikant besser war als bei Patient*innen mit konventioneller HD (148). Diese positiven Effekte auf die HRQOL konnten in der FREEDOM (Following Rehabilitation, Economics and Everyday-Dialysis Outcome Measurements) Studie bestätigt werden. Patient*innen mit kurzer täglicher Heim-HD erholten sich schneller nach den Dialysesitzungen als Patient*innen mit konventioneller Zentrums-basierter HD (149).

Aktuell gibt es keine Studie, die einen negativen Einfluss der intensivierten HD auf die HRQOL feststellt. Obwohl die Patient*innen mehr Zeit an der Dialyse verbringen, ist das Wohlbefinden im Dialyse-freien Intervall, sicherlich auch bedingt durch die kürzeren Rekonvaleszenz-Zeiten, deutlich gebessert. Diese Aspekte sollten den Patient*innen und ihrem betreuenden Umfeld im Aufklärungsgespräch über intensivierte HD-Verfahren erläutert werden, da Patient*innen den längeren Dialysezeiten zunächst skeptisch gegenüberstehen können.

\subsection{Vor- und Nachteile der einzelnen intensivierten HD-Verfahren}

Intensivierte HD-Verfahren stellen eine Möglichkeit der NET dar. Das behandelnde Team aus Nephrolog*innen, Pflegekräften, Psycholog*innen und Sozialarbeiter*innen sollte mit dem oder der Patient*in und den Angehörigen das am besten geeignete Dialyseverfahren ermitteln. Hierfür sollten die Patient*innen in einem frühen Stadium der CNI in einem nephrologischen Zentrum betreut werden, das möglichst alle Formen der NET kennt und anbietet (81). Die Patient*innen und ihre Angehörigen sollten über die verschiedenen Modalitäten der Dialyse, HD und PD, inklusive Heim-Dialyseverfahren, der Nierentransplantation inklusive Lebendspende, und je nach Alter und Komorbiditäten über eine konservative Therapie unter Verzicht einer NET aufgeklärt werden (81). Im aktuellen Dialysestandard der DGfN sind intensivierte Dialyseverfahren als Option nicht erwähnt. Im Gegensatz zur aktuellen Leitlinie der KDOQI, in der Patient*innen im Prädialysestadium auch über die Möglichkeit intensivierter HD-Verfahren aufgeklärt werden sollen (25).

Welche Form der intensivierten HD durchgeführt werden sollte, hängt von den Präferenzen des oder der Patient*in und des persönlichen Umfeldes, von medizinischen Faktoren und von dem Angebot des Dialysezentrums ab.

\section{Nächtlich intermittierende HD}

Die nächtlich intermittierende HD kann zu Hause oder im Zentrum durchgeführt werden. Anhand der beiden durchgeführten Umfragen zeigte sich, dass in den meisten Dialysezentren die nächtlich intermittierende HD unter limited care Bedingungen als zentralisierte Heimdialyse durchgeführt wird $(123,124)$. Der Vorteil dieses Verfahrens liegt darin, dass die Frequenz der 
Dialysesitzungen nicht erhöht wird und somit keine negativen Effekte auf den Gefäßzugang zu erwarten sind. Vor allem für Patient*innen, die an der Dialyse einen nahezu ungestörten Schlaf haben, stellt dies eine geeignete Option dar. Die intermittierend nächtliche HD schränkt Dialysepatient*innen am wenigsten hinsichtlich ihrer Ausbildung oder Berufsausübung ein. So eignet sie sich gerade auch für ältere Kinder und Jugendliche, wie im Berliner Nachtdialyseprogramm gezeigt werden konnte. Die Schulfehltage waren deutlich geringer als mit konventioneller HD oder PD $(120,122)$. Die Schule konnte jeden Tag im vollen Umfang besucht werden. Bei konventioneller HD am Nachmittag muss der Schulbesuch häufig vorzeitig beendet werden. Die medizinische Verantwortung des familiären Umfeldes ist bei Zentrums-basierter HD deutlich geringer als bei Heimdialyseverfahren. Nächtlich intermittierende HD kann allerdings von Dialysepatient*innen auch als belastend empfunden werden. Ok et al. beobachteten, dass $19 \%$ der Patient*innen, die initial mit nächtlich intermittierender HD behandelt wurden, die Studie abbrachen, da sie das Verfahren als unkomfortabel empfanden (111). Im Berliner Nachtdialyseprogramm haben nur zwei Jugendliche auf eigenen Wunsch zur konventionellen HD zurück gewechselt, da sie nachts an der Dialyse nicht schlafen konnten.

Die renale Restfunktion gemessen anhand der Urinmenge blieb bei nächtlich intermittierender HD gleich gut erhalten wie bei PD (122). Dies ist insoweit bemerkenswert, da die HD im Allgemeinen mit einem schnelleren Verlust der renalen Funktion im Vergleich zur PD einhergeht (36). Durch die geringeren UF-Raten bei intensivierter HD treten wahrscheinlich weniger hypotensive Episoden auf als bei konventioneller HD, was letztendlich zum Schutz der renalen Restfunktion dient (150) und die Morbidität verbessert (55).

\section{Kurze tägliche HD}

Für Patient*innen, die hohe UF-Raten schlecht tolerieren, sind tägliche intensivierte HDVerfahren, insbesondere die mit kurzer Dialysezeit, eine bedenkenswerte Option. Im pädiatrischen Bereich ist die Zentrums-basierte kurze tägliche HD vor allem für Säuglinge und Kleinkinder geeignet. Zum einen benötigt diese Altersgruppe meist eine hohe UF, da ein Großteil der Nahrung in flüssiger Form zugeführt wird, zum anderen tolerieren kleine Kinder meist keine längeren Dialysezeiten aufgrund der Einschränkung ihrer Mobilität während der Dialysesitzungen. Ebenso kann das häusliche Umfeld hierdurch entlastet werden, da ein Teil der Medikamente, wie die tägliche subkutane Injektion von Wachstumshormon, und Nahrung über eine PEG-Sonde während der Dialysesitzungen verabreicht werden können.

Allerdings scheint die tägliche HD im Zentrum für viele Patient ${ }^{\star}$ innen doch eine hohe Belastung darzustellen. Im Short Daily Trial hatten nur 78\% der Patient*innen mit kurzer täglicher HD >80\% der geforderten HD-Sitzungen (97). Zudem ging die häufigere Punktion mit einer höheren Komplikationsrate hinsichtlich des Shuntes einher (106). Achinger et al. konnten diese Unterschiede hinsichtlich der Shunt-Komplikationen unter häufigeren HD-Sitzungen allerdings nicht bestätigen (151). 
Die beiden Umfragen unter Erwachsenennphrolog*innen und Kindernephrolog*innen zeigten, dass in der gegenwärtigen Praxis die kurze tägliche HD im Zentrum häufig nicht als primäres HDVerfahren sondern als Intensivierungsmaßnahme durchgeführt wird, wenn mit konventioneller HD der oder die Patient*in unzureichend dialysiert ist $(123,124)$.

Im Heimdialyseverfahren stellt die kurze tägliche HD für Patient*innen eine Option dar, die z.B. bei nächtlichen Verfahren Schlafprobleme haben und dennoch nicht auf die Vorteile der intensivierten Heimdialyse verzichten möchten. Auch als assistiertes Verfahren in Pflegeeinrichtungen ist sie eine denkbare Option.

\section{$\underline{\text { Täglich nächtliche HD }}$}

Im Nocturnal Trial zeigten sich ebenfalls die Belastung, die tägliche HD-Sitzungen für den oder die Patient*in darstellen können: $25 \%$ der Patient*innen hatten weniger als fünf Dialysesitzungen pro Woche (98). Hinzu kommt die Belastung, die das häusliche Umfeld durch die täglich nächtliche Heim-HD erfährt (152). Rocco et al. konnten ebenso wie im Short Daily Trial eine tendenziell erhöhte, allerdings nicht signifikante Shuntkomplikationsrate mit täglich nächtlicher HD beobachten (106). Für Kinder und Jugendliche, die weite Wege zum Dialysezentrum haben, ist die nächtliche Heim-HD eine gute Option (117).

Im Nocturnal Trial wurde beobachtet, dass die renale Restfunktion mit täglich nächtlicher HD deutlich häufiger verloren ging als mit konventioneller HD. 67\% der Patient*innen mit täglich nächtlicher HD hatten nach einem Jahr keine Urinausscheidung mehr im Vergleich zu $36 \%$ der Patient*innen mit konventioneller HD (108). Letztendlich ist die Ursache des schnelleren Verlustes der renalen Restfunktion nicht geklärt und sollte in weiteren Studien evaluiert werden. Aktuell empfiehlt die KDOQI, Patient*innen über diesen möglicherweise schnelleren Verlust der renalen Restfunktion aufzuklären (25).

\subsection{Einsatz von intensivierten HD-Verfahren in der gegenwärtigen Praxis}

Über die Anwendung intensivierter Dialyseverfahren in der Routineversorgung gibt es keine Daten. Um zu analysieren, welche Einstellung Nephrolog*innen aktuell zu intensivierten HDVerfahren haben, wie viele Zentren diese Verfahren anwenden und welche inhaltlichen Einwände bzw. strukturellen und finanziellen Hindernisse für den Einsatz intensivierter HD-Verfahren gesehen werden, wurden zwei Umfragen mittels online Fragebogen durchgeführt.

In der ersten Untersuchung wurden Kindernephrolog*innen weltweit, die am International Pediatric Dialysis Network beteiligt sind, befragt (123). 69\% der Zentren boten kurze tägliche HD an; 24\% nächtlich intermittierende HD und 21\% täglich nächtliche HD. Die intensivierte HD wurde in der Hälfte der Zentren Patient*innen angeboten, die mit konventioneller HD unzureichend 
therapiert waren. Nur 17\% der Zentren boten intensivierte Verfahren im Aufklärungsgespräch zur NET ihren Patient*innen an.

In der zweiten Analyse wurden die Mitglieder der DGfN befragt (124). Fast die Hälfte der Dialysezentren, vor allem größere Zentren, boten intensivierte HD-Verfahren an. Am häufigsten wurde nächtlich intermittierende HD (72\%) gefolgt von kurzer täglicher HD (34\%) und täglich nächtlicher HD (5\%) durchgeführt. 30\% der Zentren betreuten Patient*innen mit intensivierter Heim-HD. Auch bei Erwachsenen erfolgte das Angebot intensivierter HD-Verfahren in der Hälfte der Zentren bei den Patient*innen, die mit konventioneller HD unzureichend therapiert waren.

In beiden Umfragen wurde die Frage gestellt, ob es ausreichend Evidenz gibt, intensivierte HDVerfahren gegenüber den konventionellen Verfahren zu bevorzugen. $69 \%$ der Kindernephrolog*innen und 44\% der Erwachsenennephrolog*innen sahen die Evidenz anhand der aktuellen Datenlage als gegeben. Diese Einstellung war unabhängig davon, ob in dem Zentrum, in dem sie arbeiteten, diese intensivierten Verfahren angeboten wurden oder nicht. Haupthindernisse bei der Implementierung intensivierter HD-Verfahren waren in beiden Umfragen mangelnde Vergütung und fehlendes Personal, insbesondere Dialysepflegekräfte.

Diese beiden Umfragen sind die ersten Erhebungen über den Einsatz intensivierter HD-Verfahren in der alltäglichen Praxis. Bisher publizierte Studien, vor allem im Bereich der Kindernephrologie, sind in wenigen einzelnen Zentren durchgeführt worden. Der hohe Anteil an Dialysezentren, die bereits intensivierte HD-Verfahren durchführten, entspricht sicher nicht der tatsächlichen Verbreitung intensivierter HD-Verfahren. Dialysezentren, die Interesse an intensivierten Verfahren hatten oder diese bereits durchführten, antworteten wahrscheinlich häufiger als Zentren, die kein Interesse an diesen Verfahren haben.

Obwohl die wissenschaftliche Datenlage sowohl von Kindernephrolog*innen als auch von Erwachsenennephrolog*innen überwiegend als gut eingestuft wurde, werden die Verfahren sehr selten im Aufklärungsgespräch zur NET angeboten. Der Hauptgrund ist wahrscheinlich der finanzielle Nachteil, den die Zentren erwarten, wenn mehr Patient*innen ein intensiviertes HDVerfahren erhalten würden.

\subsection{Assistierte Dialyseverfahren}

Die Durchführung einer Dialysebehandlung zu Hause kann für die Betroffenen eine schwere Belastung bedeuten. Die Patient*innen und ihre Angehörigen fühlen sich der Verantwortung für die Dialyse nicht immer gewachsen. Eine Möglichkeit stellen hierfür assistierte Dialyseverfahren dar. Assistiert kann sowohl die PD als auch die HD zu Hause durchgeführt werden. Bei den assistierten Verfahren kann der Grad der Assistenz (Auf- und Abbau der Maschinen, An- und Abschluss, kontinuierliche Überwachung) für jeden Einzelnen variiert werden. Die Assistenz durch einen Pflegedienst gerade in der Anfangszeit der Dialysebehandlung kann für viele, nicht nur für ältere Patient*innen und die Angehörigen eine Erleichterung darstellen. 
Wie viele Dialysezentren in Deutschland assistierte Dialyseverfahren anbieten, ist bislang nicht bekannt. Um zu analysieren, in welcher Form assistierte Dialyseverfahren gegenwärtig angeboten werden, führten wir eine Umfrage innerhalb der DGfN durch (125).

93\% der Befragten sahen assistierte Heim-PD und 68\% der Befragten die assistierte Heim-HD als sinnvolle Option. 55\% der Zentren boten assistierte Heimdialyse-Verfahren an (vor allem PD). Die wichtigsten Hindernisse für das Angebot assistierter Heimdialyse waren fehlende adäquate Finanzierung und Personalmangel. Assistierte Heim-HD wurde nur in 25\% der antwortenden Zentren angeboten, obwohl die Vorteile der Heim-HD in vielen Studien gezeigt werden konnten (153-155). Komplikationen mit dem Gefäßzugang waren nicht höher als bei der Zentrumsbasierten HD (154). Kardiovaskuläre Parameter, die Lebensqualität und das Überleben waren besser im Vergleich zur konventioneller Zentrums-basierten HD (155).

\subsection{Finanzierung der Dialyseverfahren}

Das größte Hindernis für den Einsatz sowohl von intensivierten als von assistierten Verfahren stellt die inadäquate Finanzierung dar (123-125).

Die Vergütung der Dialyse erfolgt in Deutschland gemäß dem einheitlichen Bewertungsmaßstab (EBM) als Dialysesachkostenpauschale (156). Diese beinhaltet alle Sachkosten, einschließlich Dialysegerät, Dialysator, Schlauchsysteme, Infusionslösungen, am Dialysetag verabreichte Heparine, Aufbereitungs- und Entsorgungsmaßnahmen, Sprechstundenbedarf sowie die Kosten der Beköstigung der Patient*innen. Weiterhin ist im Falle der HD als Heimdialyse von der Dialyseeinrichtung die Erstattung der dialysebedingten Strom-, Wasser- und Entsorgungskosten sicherzustellen.

Bei Patient*innen ab dem vollendeten 18. Lebensjahr kann die Dialysesachkostenpauschale unabhängig von der Dialysezeit pauschal für eine Dialysewoche abgerechnet werden. Für eine konventionelle HD erhält das Dialysezentrum somit die gleiche Vergütung pro Woche wie für intensivierte HD-Verfahren mit verlängerter Sitzungszeit und/oder erhöhter Frequenz (156). Aufgrund dieser Regelung sind die intensivierten HD-Verfahren für das Dialysezentrum nicht lukrativ.

Bei Kindern und Jugendlichen kann jede Dialysesitzung nach EBM einzeln abgerechnet werden, so dass die kurze tägliche HD adäquat vergütet wird, nicht aber die intensivierten Dialyseverfahren mit verlängerter Dialysezeit wie die Zentrums-basierte intermittierende nächtliche HD. Obwohl hierbei eine Dialysesitzung über zwei Kalendertage geht, zählt sie als eine Sitzung, da zwischenzeitlich kein Abschluss erfolgt. Sowohl die Sachkosten als auch die Lohnkosten des Pflegepersonals inklusive Nachtzuschläge werden nicht adäquat vergütet, so dass die zentralisierte Heimdialyse über Nacht für das Dialysezentrum mit einem finanziellen Nachtteil einhergeht. 
Bei Erwachsenen werden Heimdialyseverfahren (PD und HD) mit einer Sachkostenpauschale pro Dialysewoche vergütet. Eine Intensivierung ist auch hier für das behandelnde Dialysezentrum aus finanzieller Sicht von Nachteil. Bei Kinder und Jugendlichen gibt es für die PD ebenfalls eine Sachkostenpauschale pro Woche. Für die Heim-HD bei Patient*innen unter 18 Jahren existiert keine Abrechnungsziffer. Meist wird, wie bei Erwachsenen, die PD-Sachkostenpauschale genommen. Die Gesamtkosten für die Heim-HD liegen niedriger als bei der Zentrums-basierten HD $(117,157,158)$. Zwar ist die Heim-HD initial teurer bedingt durch Geräteanschaffung, Umbaumaßnahmen und Training, diese Kosten sind aber im Mittel nach 14 Monaten Dialysezeit ausgeglichen. Danach ist die Heim-HD finanziell für die Krankenkassen und letztendlich für die Allgemeinheit günstiger (158). Die Kostenersparnis gilt sowohl für Kinder und Jugendliche als auch für Erwachsene, auch wenn man intensivierte Heim-HD-Verfahren wie kurze tägliche HD oder nächtliche HD mit konventioneller Zentrums-basierter HD vergleicht $(117,157)$.

Für die assistierten Dialyseverfahren gibt es in vielen Ländern, wie auch in Deutschland, keine einheitliche Finanzierung von Seiten der Krankenkassen. Das bedeutet, dass die Finanzierung für jeden Einzelfall neu beantragt werden muss. In einigen Bundesländern wie in Berlin und Brandenburg haben sich Versorgungsnetzwerke gebildet, in denen Pflegedienste, Dialysezentren und die Industrie zusammenarbeiten, um die assistierte Dialyse Patient*innen anbieten zu können (159). Für die assistierte Heim-PD konnte gezeigt werden, dass sie günstiger ist als die Zentrums-basierte HD $(160,161)$.

Nach den Ergebnissen der beiden Umfragen unter Kindernephrolog*innen und Erwachsenennephrolog*innen stellt die unzureichende Finanzierung der intensivierten HDVerfahren das Haupthindernis für die flächendeckende Verbreitung dieser Verfahren dar $(123,124)$. Die kurze tägliche HD könnte bei erwachsenen Dialysepatient*innen nach einem Modell Abrechnung je Einzeldialyse, wie es pädiatrischen Patient*innen möglich ist, adäquat vergütet werden. Für die nächtliche zentralisierte Heimdialyse müsste eine gesonderte Abrechnungsziffer eingeführt werden.

Mit nächtlich zentralisierter Heimdialyse können zum einen Medikamente wie Phosphatbinder, Kaliumbinder und Antihypertensiva eingespart werden (120-122). Zum anderen sind die Patient*innen weniger Tage im Krankenhaus (122). Dialysepatient*innen mit nächtlichen Verfahren können eher eine Ausbildung absolvieren bzw. ihren Beruf ausüben. Sie benötigen daher langfristig weniger Sozialleistungen bzw. können selbst in die Sozialkassen einzahlen. Daher wäre trotz einer adäquaten Sachkostenpauschale für die nächtlich zentralisierte Heimdialyse eine langfristige Kostenersparnis zu erreichen. Hierzu sind bislang keine Kostenanalysen publiziert. 


\section{Zusammenfassung}

Das Berliner Nachtdialyse Programm für Kinder und Jugendliche in Form einer Zentrumsbasierten nächtlichen HD wurde vor über zehn Jahren initiiert. Die positiven Effekte der intensivierten HD, wie sie vor allem bei Erwachsenen schon beschrieben waren, konnten bestätigt werden. Mit intermittierend nächtlicher HD verbesserte sich die kardiovaskuläre Morbidität deutlich. Der arterielle Blutdruck und die Serumphosphatspiegel waren signifikant niedriger. Flüssigkeits- und diätetische Restriktionen konnten im Vergleich zu konventionellen Dialyseverfahren wie HD und PD aufgehoben werden. Durch die Verlagerung der Dialyse in die Nachtstunden konnten die Patient*innen in vollem Umfang ihrer schulischen Ausbildung und ihren Freizeitaktivitäten nachkommen bei zugleich besserer medizinischer Versorgung.

Im Weiteren wurde der Einsatz individueller, sowohl intensivierter als auch assistierter, Dialyseverfahren bei Kindern, Jugendlichen und Erwachsenen in der gegenwärtigen Praxis untersucht. Inadäquate Finanzierung stellte das Haupthindernis für die flächendeckende Verbreitung individualisierter Dialyseverfahren dar.

Die Motivation für den Einsatz individualisierter Dialyseverfahren ist die Lebensqualität und Morbidität der Dialysepatient*innen unabhängig ihres Lebensalters zu verbessern. Die Möglichkeit für junge Patient*innen bei nächtlichen Verfahren am normalen Berufs- und Schulalltag teilnehmen zu können, verbessern die Rehabilitationsaussichten erheblich. 


\section{Literaturangaben}

1. ERA-EDTA Registry: annual reports [Internet]. [zitiert 28. September 2016]. Verfügbar unter: https://www.era-edta-reg.org/index.jsp?p=14

2. Ploos van Amstel S, Noordzij M, Warady BA, Cano F, Craig JC, Groothoff JW, u. a. Renal replacement therapy for children throughout the world: the need for a global registry. Pediatr Nephrol Berl Ger. Mai 2018;33(5):863-71.

3. Ehrich JH, Loirat C, Brunner FP, Geerlings W, Landais P, Mallick NP, u. a. Report on management of renal failure in children in Europe, XXII, 1991. Nephrol Dial Transplant Off Publ Eur Dial Transpl Assoc - Eur Ren Assoc. 1992;7 Suppl 2:36-48.

4. Chesnaye NC, Schaefer F, Groothoff JW, Bonthuis M, Reusz G, Heaf JG, u. a. Mortality risk in European children with end-stage renal disease on dialysis. Kidney Int. 2016;89(6):135562.

5. Oh J, Wunsch R, Turzer M, Bahner M, Raggi P, Querfeld U, u. a. Advanced coronary and carotid arteriopathy in young adults with childhood-onset chronic renal failure. Circulation. 2. Juli 2002;106(1):100-5.

6. Mitsnefes MM. Cardiovascular disease in children with chronic kidney disease. J Am Soc Nephrol JASN. April 2012;23(4):578-85.

7. Kramer A, Stel VS, Tizard J, Verrina E, Rönnholm K, Pálsson R, u. a. Characteristics and survival of young adults who started renal replacement therapy during childhood. Nephrol Dial Transplant Off Publ Eur Dial Transpl Assoc - Eur Ren Assoc. März 2009;24(3):926-33.

8. Brancaccio D, Bellasi A, Cozzolino M, Galassi A, Gallieni M. Arterial accelerated aging in dialysis patients: the clinical impact of vascular calcification. Curr Vasc Pharmacol. Juli 2009;7(3):374-80.

9. Splinter A, Tjaden LA, Haverman L, Adams B, Collard L, Cransberg K, u. a. Children on dialysis as well as renal transplanted children report severely impaired health-related quality of life. Qual Life Res Int J Qual Life Asp Treat Care Rehabil. Juni 2018;27(6):1445-54.

10. Tjaden LA, Grootenhuis MA, Noordzij M, Groothoff JW. Health-related quality of life in patients with pediatric onset of end-stage renal disease: state of the art and recommendations for clinical practice. Pediatr Nephrol Berl Ger. 2016;31(10):1579-91.

11. Soliman NA, Ali RI, Ghobrial EE, Habib EI, Ziada AM. Pattern of clinical presentation of congenital anomalies of the kidney and urinary tract among infants and children. Nephrology. Juni 2015;20(6):413-8.

12. Bulum B, Özçakar ZB, Üstüner E, Düşünceli E, Kavaz A, Duman D, u. a. High frequency of kidney and urinary tract anomalies in asymptomatic first-degree relatives of patients with CAKUT. Pediatr Nephrol. 28. November 2013;28(11):2143-7.

13. Loane M, Dolk H, Kelly A, Teljeur C, Greenlees R, Densem J, u. a. Paper 4: EUROCAT statistical monitoring: identification and investigation of ten year trends of congenital anomalies in Europe. Birt Defects Res A Clin Mol Teratol. März 2011;91 Suppl 1:S31-43.

14. Caruana G, Bertram JF. Congenital anomalies of the kidney and urinary tract genetics in mice and men. Nephrol Carlton Vic. Mai 2015;20(5):309-11.

15. Nicolaou N, Renkema KY, Bongers EMHF, Giles RH, Knoers NVAM. Genetic, environmental, and epigenetic factors involved in CAKUT. Nat Rev Nephrol. Dezember 2015;11(12):72031. 
16. Wühl E, van Stralen KJ, Wanner C, Ariceta G, Heaf JG, Bjerre AK, u. a. Renal replacement therapy for rare diseases affecting the kidney: an analysis of the ERA-EDTA Registry. Nephrol Dial Transplant Off Publ Eur Dial Transpl Assoc - Eur Ren Assoc. September 2014;29 Suppl 4:iv1-8.

17. Hoyer PF. Clinical manifestations of autosomal recessive polycystic kidney disease. Curr Opin Pediatr. April 2015;27(2):186-92.

18. Braun DA, Hildebrandt F. Ciliopathies. Cold Spring Harb Perspect Biol. 1. März 2017;9(3).

19. König JC, Titieni A, Konrad M, NEOCYST Consortium. Network for Early Onset Cystic Kidney Diseases-A Comprehensive Multidisciplinary Approach to Hereditary Cystic Kidney Diseases in Childhood. Front Pediatr. 2018;6:24.

20. Wehrman A, Kriegermeier A, Wen J. Diagnosis and Management of Hepatobiliary Complications in Autosomal Recessive Polycystic Kidney Disease. Front Pediatr. 2017;5:124.

21. Mirvis M, Stearns T, James Nelson W. Cilium structure, assembly, and disassembly regulated by the cytoskeleton. Biochem J. 31. Juli 2018;475(14):2329-53.

22. Habbig S, Liebau MC. Ciliopathies - from rare inherited cystic kidney diseases to basic cellular function. Mol Cell Pediatr. Dezember 2015;2(1):8.

23. Trautmann A, Bodria M, Ozaltin F, Gheisari A, Melk A, Azocar M, u. a. Spectrum of steroidresistant and congenital nephrotic syndrome in children: the PodoNet registry cohort. Clin J Am Soc Nephrol CJASN. 7. April 2015;10(4):592-600.

24. Rheault MN, Gbadegesin RA. The Genetics of Nephrotic Syndrome. J Pediatr Genet. März 2016;5(1):15-24.

25. Daugirdas JT, Depner TA, Inrig J, Mehrotra R, Rocco MV, Suri RS, u. a. KDOQI Clinical Practice Guideline for Hemodialysis Adequacy: 2015 Update. Am J Kidney Dis. 1. November 2015;66(5):884-930.

26. Harambat J, van Stralen KJ, Schaefer F, Grenda R, Jankauskiene A, Kostic M, u. a. Disparities in policies, practices and rates of pediatric kidney transplantation in Europe. Am J Transplant Off J Am Soc Transplant Am Soc Transpl Surg. August 2013;13(8):2066-74.

27. Schwartz GJ, Muñoz A, Schneider MF, Mak RH, Kaskel F, Warady BA, u. a. New equations to estimate GFR in children with CKD. J Am Soc Nephrol JASN. März 2009;20(3):629-37.

28. Filler G, Lepage N. Should the Schwartz formula for estimation of GFR be replaced by cystatin C formula? Pediatr Nephrol Berl Ger. Oktober 2003;18(10):981-5.

29. National Kidney Foundation. KDOQI Clinical Practice Guidelines and Clinical Practice Recommendations for 2006 Updates. Hemodialysis Adequacy, Peritoneal Dialysis Adequacy, Vascular Access [Internet]. [zitiert 16. Januar 2019]. Verfügbar unter: http://www.kidney.org/professionals/KDOQI/guideline_upHD_PD_VA/index.htm.

30. Cooper BA, Branley P, Bulfone L, Collins JF, Craig JC, Fraenkel MB, u. a. A randomized, controlled trial of early versus late initiation of dialysis. $\mathrm{N}$ Engl $\mathrm{J}$ Med. 12. August 2010;363(7):609-19.

31. Bakkaloğlu SA, Kandur Y, Serdaroğlu E, Noyan A, Bayazıt AK, Sever L, u. a. Effect of the timing of dialysis initiation on left ventricular hypertrophy and Inflammation in pediatric patients. Pediatr Nephrol Berl Ger. September 2017;32(9):1595-602. 
32. Saban JA, Zappitelli M, Samuel SM, Sood MM, Alexander RT, Arora S, u. a. Perceptions of pediatric nephrologists regarding timing of dialysis initiation in children in Canada. Can $\mathrm{J}$ Kidney Health Dis. 2016;3:31.

33. Renal Physicians Association. Shared decision-making in the appropriate initiation of and withdrawal from dialysis: Clinical practice Guideline Recommendation Summary. Second edition. RPA, Rockville [Internet]. [zitiert 16. Januar 2019] Verfügbar unter: https://www.renalmd.org.

34. Dionne JM, d'Agincourt-Canning L. Sustaining life or prolonging dying? Appropriate choice of conservative care for children in end-stage renal disease: an ethical framework. Pediatr Nephrol Berl Ger. Oktober 2015;30(10):1761-9.

35. Chesnaye N, Bonthuis M, Schaefer F, Groothoff JW, Verrina E, Heaf JG, u. a. Demographics of paediatric renal replacement therapy in Europe: a report of the ESPN/ERA-EDTA registry. Pediatr Nephrol Berl Ger. Dezember 2014;29(12):2403-10.

36. Roszkowska-Blaim M, Skrzypczyk P, Jander A, Tkaczyk M, Bałasz-Chmielewska I, Zurowska $A, u$. a. The effect of peritoneal dialysis method on residual renal function in children. Adv Perit Dial Conf Perit Dial. 2012;28:112-9.

37. Warady BA, Bakkaloglu S, Newland J, Cantwell M, Verrina E, Neu A, u. a. Consensus guidelines for the prevention and treatment of catheter-related infections and peritonitis in pediatric patients receiving peritoneal dialysis: 2012 update. Perit Dial Int J Int Soc Perit Dial. Juni 2012;32 Suppl 2:S32-86.

38. Kiliś-Pstrusińska K, Wasilewska A, Medyńska A, Bałasz-Chmielewska I, Grenda R, KluskaJóźwiak A, u. a. Psychosocial aspects of children and families of children treated with automated peritoneal dialysis. Pediatr Nephrol Berl Ger. November 2013;28(11):2157-67.

39. Schaefer F, Warady BA. Peritoneal dialysis in children with end-stage renal disease. Nat Rev Nephrol. November 2011;7(11):659-68.

40. Vidal E, Chesnaye NC, Paglialonga F, Minale B, Leozappa G, Giordano M, u. a. A propensitymatched comparison of hard outcomes in children on chronic dialysis. Eur J Pediatr. Januar 2018;177(1):117-24.

41. Hayes WN, Watson AR, Callaghan N, Wright E, Stefanidis CJ, European Pediatric Dialysis Working Group. Vascular access: choice and complications in European paediatric haemodialysis units. Pediatr Nephrol Berl Ger. Juni 2012;27(6):999-1004.

42. Almási-Sperling V, Galiano M, Lang W, Rother U, Rascher W, Regus S. Timing of first arteriovenous fistula cannulation in children on hemodialysis. Pediatr Nephrol Berl Ger. 2016;31(10):1647-57.

43. Zaritsky JJ, Salusky IB, Gales B, Ramos G, Atkinson J, Allsteadt A, u. a. Vascular access complications in long-term pediatric hemodialysis patients. Pediatr Nephrol Berl Ger. November 2008;23(11):2061-5.

44. Quinlan C, Bates M, Sheils A, Dolan N, Riordan M, Awan A. Chronic hemodialysis in children weighing less than $10 \mathrm{~kg}$. Pediatr Nephrol Berl Ger. Mai 2013;28(5):803-9.

45. Daugirdas JT. Second generation logarithmic estimates of single-pool variable volume Kt/V: an analysis of error. J Am Soc Nephrol JASN. November 1993;4(5):1205-13.

46. Cano F, Azocar M, Cavada G, Delucchi A, Marin V, Rodriguez E. Kt/V and nPNA in pediatric peritoneal dialysis: a clinical or a mathematical association? Pediatr Nephrol Berl Ger. Januar 2006;21(1):114-8. 
47. Hörl WH. Dialyseverfahren in Klinik und Praxis: Technik und Klinik. 6. Auflage, Georg Thieme Verlag; 2004. 714 S.

48. Saran R, Bragg-Gresham JL, Levin NW, Twardowski ZJ, Wizemann V, Saito A, u. a. Longer treatment time and slower ultrafiltration in hemodialysis: associations with reduced mortality in the DOPPS. Kidney Int. April 2006;69(7):1222-8.

49. Goldstein SL. Adequacy of dialysis in children: does small solute clearance really matter? Pediatr Nephrol Berl Ger. Januar 2004;19(1):1-5.

50. Shafi T, Jaar BG, Plantinga LC, Fink NE, Sadler JH, Parekh RS, u. a. Association of residual urine output with mortality, quality of life, and inflammation in incident hemodialysis patients: the Choices for Healthy Outcomes in Caring for End-Stage Renal Disease (CHOICE) Study. Am J Kidney Dis Off J Natl Kidney Found. August 2010;56(2):348-58.

51. Obi Y, Rhee CM, Mathew AT, Shah G, Streja E, Brunelli SM, u. a. Residual Kidney Function Decline and Mortality in Incident Hemodialysis Patients. J Am Soc Nephrol JASN. Dezember 2016;27(12):3758-68.

52. Marquez IO, Tambra S, Luo FY, Li Y, Plummer NS, Hostetter TH, u. a. Contribution of Residual Function to Removal of Protein-Bound Solutes in Hemodialysis. Clin J Am Soc Nephrol. 2. Januar 2011;6(2):290-6.

53. Bammens B, Evenepoel P, Verbeke K, Vanrenterghem Y. Removal of middle molecules and protein-bound solutes by peritoneal dialysis and relation with uremic symptoms. Kidney Int. Dezember 2003;64(6):2238-43.

54. Wang AY-M, Lam CW-K, Wang M, Woo J, Chan IH-S, Lui S-F, u. a. Circulating soluble vascular cell adhesion molecule 1: Relationships with residual renal function, cardiac hypertrophy, and outcome of peritoneal dialysis patients. Am J Kidney Dis. 1. April 2005;45(4):715-29.

55. Bargman JM, Thorpe KE, Churchill DN, CANUSA Peritoneal Dialysis Study Group. Relative contribution of residual renal function and peritoneal clearance to adequacy of dialysis: a reanalysis of the CANUSA study. J Am Soc Nephrol JASN. Oktober 2001;12(10):2158-62.

56. Johansen KL, Chertow GM, Kutner NG, Dalrymple LS, Grimes BA, Kaysen GA. Low level of self-reported physical activity in ambulatory patients new to dialysis. Kidney Int. Dezember 2010;78(11):1164-70.

57. Murtagh FEM, Addington-Hall J, Higginson IJ. The prevalence of symptoms in end-stage renal disease: a systematic review. Adv Chronic Kidney Dis. Januar 2007;14(1):82-99.

58. Rees L. Assessment of dialysis adequacy: beyond urea kinetic measurements. Pediatr Nephrol Berl Ger. Januar 2019;34(1):61-9.

59. Stevens KK, Denby L, Patel RK, Mark PB, Kettlewell S, Smith GL, u. a. Deleterious effects of phosphate on vascular and endothelial function via disruption to the nitric oxide pathway. Nephrol Dial Transplant Off Publ Eur Dial Transpl Assoc - Eur Ren Assoc. 1. Oktober 2017;32(10):1617-27.

60. Aleksova J, Kurniawan S, Vucak-Dzumhur M, Kerr P, Ebeling PR, Milat F, u. a. Aortic vascular calcification is inversely associated with the trabecular bone score in patients receiving dialysis. Bone. August 2018;113:118-23.

61. Jankowska M, Cobo G, Lindholm B, Stenvinkel P. Inflammation and Protein-Energy Wasting in the Uremic Milieu. Expand Hemodial. 2017;191:58-71. 
62. Ruderman I, Holt SG, Hewitson TD, Smith ER, Toussaint ND. Current and potential therapeutic strategies for the management of vascular calcification in patients with chronic kidney disease including those on dialysis. Semin Dial. September 2018;31(5):487-99.

63. Shroff R, Quinlan C, Mitsnefes M. Uraemic vasculopathy in children with chronic kidney disease: prevention or damage limitation? Pediatr Nephrol Berl Ger. Juni 2011;26(6):85365.

64. Shroff R. Phosphate is a vascular toxin. Pediatr Nephrol Berl Ger. April 2013;28(4):583-93.

65. Sethna CB, Merchant K, Reyes A. Cardiovascular Disease Risk in Children With Kidney Disease. Semin Nephrol. Mai 2018;38(3):298-313.

66. Kramer AM, van Stralen KJ, Jager KJ, Schaefer F, Verrina E, Seeman T, u. a. Demographics of blood pressure and hypertension in children on renal replacement therapy in Europe. Kidney Int. November 2011;80(10):1092-8.

67. Civilibal M, Caliskan S, Adaletli I, Oflaz H, Sever L, Candan C, u. a. Coronary artery calcifications in children with end-stage renal disease. Pediatr Nephrol. 1. Oktober 2006;21(10):1426-33.

68. Covic A, Mardare N, Gusbeth-Tatomir P, Brumaru O, Gavrilovici C, Munteanu M, u. a. Increased arterial stiffness in children on haemodialysis. Nephrol Dial Transplant. 1. März 2006;21(3):729-35.

69. Covic A, Gusbeth-Tatomir P, Goldsmith DJA. Arterial Stiffness in Renal Patients: An Update. Am J Kidney Dis. 1. Juni 2005;45(6):965-77.

70. Purnell TS, Auguste P, Crews DC, Lamprea-Montealegre J, Olufade T, Greer R, u. a. Comparison of life participation activities among adults treated by hemodialysis, peritoneal dialysis, and kidney transplantation: a systematic review. Am J Kidney Dis Off J Natl Kidney Found. November 2013;62(5):953-73.

71. Mapes DL, Bragg-Gresham JL, Bommer J, Fukuhara S, McKevitt P, Wikström B, u. a. Healthrelated quality of life in the Dialysis Outcomes and Practice Patterns Study (DOPPS). Am J Kidney Dis Off J Natl Kidney Found. November 2004;44(5 Suppl 2):54-60.

72. van Sandwijk MS, Al Arashi D, van de Hare FM, van der Torren JMR, Kersten M-J, Bijlsma $J A$, u. a. Fatigue, anxiety, depression and quality of life in kidney transplant recipients, haemodialysis patients, patients with a haematological malignancy and healthy controls. Nephrol Dial Transplant Off Publ Eur Dial Transpl Assoc - Eur Ren Assoc. 2. Mai 2018;

73. Francis A, Didsbury MS, Zwieten A van, Chen K, James LJ, Kim S, u. a. Quality of life of children and adolescents with chronic kidney disease: a cross-sectional study. Arch Dis Child. 17. Juli 2018;archdischild-2018-314934.

74. Haffner D, Zivicnjak M. Pubertal development in children with chronic kidney disease. Pediatr Nephrol Berl Ger. 2017;32(6):949-64.

75. Harambat J, Bonthuis M, van Stralen KJ, Ariceta G, Battelino N, Bjerre A, u. a. Adult height in patients with advanced CKD requiring renal replacement therapy during childhood. Clin $\mathrm{J}$ Am Soc Nephrol CJASN. Januar 2014;9(1):92-9.

76. Daugirdas JT. Hemodialysis Treatment Time: As Important as it Seems? Semin Dial. 2017;30(2):93-8.

77. Paniagua R, Amato D, Vonesh E, Correa-Rotter R, Ramos A, Moran J, u. a. Effects of increased peritoneal clearances on mortality rates in peritoneal dialysis: ADEMEX, a prospective, randomized, controlled trial. J Am Soc Nephrol JASN. Mai 2002;13(5):1307-20. 
78. Eknoyan G, Beck GJ, Cheung AK, Daugirdas JT, Greene T, Kusek JW, u. a. Effect of dialysis dose and membrane flux in maintenance hemodialysis. $N$ Engl $J$ Med. 19. Dezember 2002;347(25):2010-9.

79. Geberth S, Nowack R. Praxis der Dialyse. 1. Auflage, Springer-Verlag; 2014. 409 S.

80. Locatelli F, Carfagna F, Del Vecchio L, La Milia V. Haemodialysis or haemodiafiltration: that is the question. Nephrol Dial Transplant Off Publ Eur Dial Transpl Assoc - Eur Ren Assoc. 1. November 2018;33(11):1896-904.

81. Dialyse-Standard - Deutsche Gesellschaft für Nephrologie [Internet]. [zitiert 5. Juni 2018]. Verfügbar unter: https://www.dgfn.eu/dialyse-standard.html

82. Grooteman MPC, van den Dorpel MA, Bots ML, Penne EL, van der Weerd NC, Mazairac AHA, u. a. Effect of online hemodiafiltration on all-cause mortality and cardiovascular outcomes. J Am Soc Nephrol JASN. Juni 2012;23(6):1087-96.

83. Ok E, Asci G, Toz H, Ok ES, Kircelli F, Yilmaz M, u. a. Mortality and cardiovascular events in online haemodiafiltration (OL-HDF) compared with high-flux dialysis: results from the Turkish OL-HDF Study. Nephrol Dial Transplant Off Publ Eur Dial Transpl Assoc - Eur Ren Assoc. Januar 2013;28(1):192-202.

84. Maduell F, Moreso F, Pons M, Ramos R, Mora-Macià J, Carreras J, u. a. High-efficiency postdilution online hemodiafiltration reduces all-cause mortality in hemodialysis patients. $J$ Am Soc Nephrol JASN. Februar 2013;24(3):487-97.

85. Nistor I, Palmer SC, Craig JC, Saglimbene V, Vecchio M, Covic A, u. a. Haemodiafiltration, haemofiltration and haemodialysis for end-stage kidney disease. Cochrane Database Syst Rev. 2015;5:CD006258.

86. Shroff R, Bayazit A, Stefanidis CJ, Askiti V, Azukaitis K, Canpolat N, u. a. Effect of haemodiafiltration vs conventional haemodialysis on growth and cardiovascular outcomes in children - the HDF, heart and height (3H) study. BMC Nephrol. 10. August 2018;19(1):199.

87. Thumfart J, Pommer W, Querfeld U, Müller D. Intensified hemodialysis in adults, and in children and adolescents. Dtsch Ärztebl Int. 4. April 2014;111(14):237-43.

88. Fadem SZ. Issues in Dialysis. 1. Auflage. Nova; 2012. 103-112 S.

89. McFarlane PA. More of the same: improving outcomes through intensive hemodialysis. Semin Dial. Dezember 2009;22(6):598-602.

90. Troidle L, Finkelstein F, Hotchkiss M, Leypoldt JK. Enhanced solute removal with intermittent, in-center, 8-hour nocturnal hemodialysis. Hemodial Int Int Symp Home Hemodial. Oktober 2009;13(4):487-91.

91. Hussein WF, Arramreddy R, Sun SJ, Reiterman M, Schiller B. Higher Ultrafiltration Rate Is Associated with Longer Dialysis Recovery Time in Patients Undergoing Conventional Hemodialysis. Am J Nephrol. 2017;46(1):3-10.

92. Yu J, Liu Z, Shen B, Teng J, Zou J, Ding X. Intradialytic Hypotension as an Independent Risk Factor for Long-Term Mortality in Maintaining Hemodialysis Patients: A 5-Year Follow-Up Cohort Study. Blood Purif. 2018;45(4):320-6.

93. Movilli E, Gaggia P, Zubani R, Camerini C, Vizzardi V, Parrinello G, u. a. Association between high ultrafiltration rates and mortality in uraemic patients on regular haemodialysis. A 5-year prospective observational multicentre study. Nephrol Dial Transplant Off Publ Eur Dial Transpl Assoc - Eur Ren Assoc. Dezember 2007;22(12):3547-52. 
94. Assimon MM, Wenger JB, Wang L, Flythe JE. Ultrafiltration Rate and Mortality in Maintenance Hemodialysis Patients. Am J Kidney Dis Off J Natl Kidney Found. Dezember 2016;68(6):911-22.

95. Hecking M, MoissI U, Genser B, Rayner H, Dasgupta I, Stuard S, u. a. Greater fluid overload and lower interdialytic weight gain are independently associated with mortality in a large international hemodialysis population. Nephrol Dial Transplant Off Publ Eur Dial Transpl Assoc - Eur Ren Assoc. 1. Oktober 2018;33(10):1832-42.

96. Charra B, Calemard E, Cuche M, Laurent G. Control of hypertension and prolonged survival on maintenance hemodialysis. Nephron. 1983;33(2):96-9.

97. FHN Trial Group, Chertow GM, Levin NW, Beck GJ, Depner TA, Eggers PW, u. a. In-center hemodialysis six times per week versus three times per week. N Engl J Med. 9. Dezember 2010;363(24):2287-300.

98. Rocco MV, Lockridge RS Jr, Beck GJ, Eggers PW, Gassman JJ, Greene T, u. a. The effects of frequent nocturnal home hemodialysis: the Frequent Hemodialysis Network Nocturnal Trial. Kidney Int. November 2011;80(10):1080-91.

99. Jardine MJ, Zuo L, Gray NA, de Zoysa JR, Chan CT, Gallagher MP, u. a. A Trial of Extending Hemodialysis Hours and Quality of Life. J Am Soc Nephrol JASN. Juni 2017;28(6):1898-911.

100. Culleton BF, Walsh M, Klarenbach SW, Mortis G, Scott-Douglas N, Quinn RR, u. a. Effect of frequent nocturnal hemodialysis vs conventional hemodialysis on left ventricular mass and quality of life: a randomized controlled trial. JAMA J Am Med Assoc. 19. September 2007;298(11):1291-9.

101. Chan CT, Chertow GM, Daugirdas JT, Greene TH, Kotanko P, Larive B, u. a. Effects of daily hemodialysis on heart rate variability: results from the Frequent Hemodialysis Network (FHN) Daily Trial. Nephrol Dial Transplant Off Publ Eur Dial Transpl Assoc - Eur Ren Assoc. Januar 2014;29(1):168-78.

102. Daugirdas JT, Chertow GM, Larive B, Pierratos A, Greene T, Ayus JC, u. a. Effects of frequent hemodialysis on measures of CKD mineral and bone disorder. J Am Soc Nephrol JASN. April 2012;23(4):727-38.

103. Chertow GM, Levin NW, Beck GJ, Daugirdas JT, Eggers PW, Kliger AS, u. a. Long-Term Effects of Frequent In-Center Hemodialysis. J Am Soc Nephrol JASN. Juni 2016;27(6):18306.

104. Hall YN, Larive B, Painter P, Kaysen GA, Lindsay RM, Nissenson AR, u. a. Effects of six versus three times per week hemodialysis on physical performance, health, and functioning: Frequent Hemodialysis Network (FHN) randomized trials. Clin J Am Soc Nephrol CJASN. Mai 2012;7(5):782-94.

105. Garg AX, Suri RS, Eggers P, Finkelstein FO, Greene T, Kimmel PL, u. a. Patients receiving frequent hemodialysis have better health-related quality of life compared to patients receiving conventional hemodialysis. Kidney Int. 2017;91(3):746-54.

106. Suri RS, Larive B, Sherer S, Eggers P, Gassman J, James SH, u. a. Risk of vascular access complications with frequent hemodialysis. J Am Soc Nephrol JASN. Februar 2013;24(3):498-505.

107. Kotanko P, Garg AX, Depner T, Pierratos A, Chan CT, Levin NW, u. a. Effects of frequent hemodialysis on blood pressure: Results from the randomized frequent hemodialysis network trials. Hemodial Int Int Symp Home Hemodial. Juli 2015;19(3):386-401.

108. Daugirdas JT, Greene T, Rocco MV, Kaysen GA, Depner TA, Levin NW, u. a. Effect of frequent hemodialysis on residual kidney function. Kidney Int. Mai 2013;83(5):949-58. 
109. Rocco MV, Daugirdas JT, Greene T, Lockridge RS, Chan C, Pierratos A, u. a. Long-term Effects of Frequent Nocturnal Hemodialysis on Mortality: The Frequent Hemodialysis Network (FHN) Nocturnal Trial. Am J Kidney Dis Off J Natl Kidney Found. September 2015;66(3):459-68.

110. USRDS. Annual Data Report 2017 [Internet]. [zitiert 7. Juni 2018]. Verfügbar unter: https://www.usrds.org/adr.aspx

111. Ok E, Duman S, Asci G, Tumuklu M, Onen Sertoz O, Kayikcioglu M, u. a. Comparison of 4- and 8-h dialysis sessions in thrice-weekly in-centre haemodialysis: a prospective, casecontrolled study. Nephrol Dial Transplant Off Publ Eur Dial Transpl Assoc - Eur Ren Assoc. April 2011;26(4):1287-96.

112. Lacson E Jr, Xu J, Suri RS, Nesrallah G, Lindsay R, Garg AX, u. a. Survival with threetimes weekly in-center nocturnal versus conventional hemodialysis. J Am Soc Nephrol JASN. April 2012;23(4):687-95.

113. Fischbach M, Terzic J, Laugel V, Dheu C, Menouer S, Helms P, u. a. Daily on-line haemodiafiltration: a pilot trial in children. Nephrol Dial Transplant Off Publ Eur Dial Transpl Assoc - Eur Ren Assoc. September 2004;19(9):2360-7.

114. Fischbach M, Terzic J, Menouer S, Dheu C, Seuge L, Zalosczic A. Daily on line haemodiafiltration promotes catch-up growth in children on chronic dialysis. Nephrol Dial Transplant Off Publ Eur Dial Transpl Assoc - Eur Ren Assoc. März 2010;25(3):867-73.

115. de Camargo MFC, Henriques CL, Vieira S, Komi S, Leão ER, Nogueira PCK. Growth of children with end-stage renal disease undergoing daily hemodialysis. Pediatr Nephrol Berl Ger. März 2014;29(3):439-44.

116. Fischbach M, Dheu C, Seuge L, Menouer S, Terzic J. In-center daily on-line hemodiafiltration: a 4-year experience in children. Clin Nephrol. April 2008;69(4):279-84.

117. Geary DF, Piva E, Tyrrell J, Gajaria MJ, Picone G, Keating LE, u. a. Home nocturnal hemodialysis in children. J Pediatr. September 2005;147(3):383-7.

118. Hothi DK, Harvey E, Piva E, Keating L, Secker D, Geary DF. Calcium and phosphate balance in adolescents on home nocturnal haemodialysis. Pediatr Nephrol Berl Ger. Juni 2006;21(6):835-41.

119. Geary DF, Piva E, Gajaria M, Tyrrel J, Picone G, Harvey E. Development of a nocturnal home hemodialysis (NHHD) program for children. Semin Dial. April 2004;17(2):115-7.

120. Hoppe A, von Puttkamer C, Linke U, Kahler C, Booss M, Braunauer-Kolberg R, u. a. A hospital-based intermittent nocturnal hemodialysis program for children and adolescents. $J$ Pediatr. Januar 2011;158(1):95-9, 99.e1.

121. Thumfart J, Puttkamer CV, Wagner S, Querfeld U, Müller D. Hemodiafiltration in a pediatric nocturnal dialysis program. Pediatr Nephrol Berl Ger. August 2014;29(8):1411-6.

122. Thumfart J, Hilliger T, Stiny C, Wagner S, Querfeld U, Müller D. Is peritoneal dialysis still an equal option? Results of the Berlin pediatric nocturnal dialysis program. Pediatr Nephrol Berl Ger. Juli 2015;30(7):1181-7.

123. Thumfart J, Müller D, Wagner S, Jayanti A, Borzych-Duzalka D, Schaefer F, u. a. Barriers for implementation of intensified hemodialysis: survey results from the International Pediatric Dialysis Network. Pediatr Nephrol Berl Ger. April 2018;33(4):705-12.

124. Thumfart J, Wagner S, Jayanti A, Müller D, Pommer W. Attitudes of nephrologists towards intensified hemodialysis. Clin Nephrol. Oktober 2018;90(4):255-61. 
125. Pommer W, Wagner S, Müller D, Thumfart J. Attitudes of nephrologists towards assisted home dialysis in Germany. Clin Kidney J. Juni 2018;11(3):400-5.

126. Weaver DJ, Somers MJG, Martz K, Mitsnefes MM. Clinical outcomes and survival in pediatric patients initiating chronic dialysis: a report of the NAPRTCS registry. Pediatr Nephrol Berl Ger. Dezember 2017;32(12):2319-30.

127. Khandelwal P, Murugan V, Hari S, Lakshmy R, Sinha A, Hari P, u. a. Dyslipidemia, carotid intima-media thickness and endothelial dysfunction in children with chronic kidney disease. Pediatr Nephrol Berl Ger. 2016;31(8):1313-20.

128. Shroff R, Weaver DJ Jr, Mitsnefes MM. Cardiovascular complications in children with chronic kidney disease. Nat Rev Nephrol. November 2011;7(11):642-9.

129. Demirci C, Ozkahya M, Demirci MS, Asci G, Kose T, Colak T, u. a. Effects of three times weekly eight-hour nocturnal hemodialysis on volume and nutritional status. Am J Nephrol. 2013;37(6):559-67.

130. Bugeja A, Dacouris N, Thomas A, Marticorena R, McFarlane P, Donnelly S, u. a. In-center nocturnal hemodialysis: another option in the management of chronic kidney disease. Clin $\mathrm{J}$ Am Soc Nephrol CJASN. April 2009;4(4):778-83.

131. David S, Kümpers P, Eisenbach GM, Haller H, Kielstein JT. Prospective evaluation of an in-centre conversion from conventional haemodialysis to an intensified nocturnal strategy. Nephrol Dial Transplant Off Publ Eur Dial Transpl Assoc - Eur Ren Assoc. Juli 2009;24(7):2232-40.

132. Troidle L, Hotchkiss M, Finkelstein F. A thrice weekly in-center nocturnal hemodialysis program. Adv Chronic Kidney Dis. Juli 2007;14(3):244-8.

133. McGregor DO, Buttimore AL, Lynn KL, Nicholls MG, Jardine DL. A Comparative Study of Blood Pressure Control with Short In-Center versus Long Home Hemodialysis. Blood Purif. 2001;19(3):293-300.

134. Jin X, Rong S, Mei C, Ye C, Chen J, Chen X. Effects of in-center nocturnal versus conventional hemodialysis on endothelial dysfunction. Ther Apher Dial Off Peer-Rev J Int Soc Apher Jpn Soc Apher Jpn Soc Dial Ther. August 2012;16(4):334-40.

135. Woods JD, Port FK, Orzol S, Buoncristiani U, Young E, Wolfe RA, u. a. Clinical and biochemical correlates of starting „daily“ hemodialysis. Kidney Int. Juni 1999;55(6):2467-76.

136. Lorenzen JM, Thum T, Eisenbach GM, Haller H, Kielstein JT. Conversion from conventional in-centre thrice-weekly haemodialysis to short daily home haemodialysis ameliorates uremia-associated clinical parameters. Int Urol Nephrol. Juni 2012;44(3):88390 .

137. Lindsay RM, Suri RS, Moist LM, Garg AX, Cuerden M, Langford S, u. a. International Quotidian Dialysis Registry: Annual report 2010. Hemodial Int Int Symp Home Hemodial. Januar 2011;15(1):15-22.

138. Bergman A, Fenton SSA, Richardson RMA, Chan CT. Reduction in cardiovascular related hospitalization with nocturnal home hemodialysis. Clin Nephrol. Januar 2008;69(1):33-9.

139. Yuen D, Pierratos A, Richardson RMA, Chan CT. The natural history of coronary calcification progression in a cohort of nocturnal haemodialysis patients. Nephrol Dial Transplant Off Publ Eur Dial Transpl Assoc - Eur Ren Assoc. Mai 2006;21(5):1407-12.

140. WHO | WHOQOL: Measuring Quality of Life [Internet]. WHO. [zitiert 24. Juni 2018]. Verfügbar unter: http://www.who.int/healthinfo/survey/whoqol-qualityoflife/en/ 
141. Daig I, Lehmann A. Verfahren zur Messung der Lebensqualität. Z Med Psychol. 2007;16:5-23.

142. Herschbach P. Das „Zufriedenheitsparadox” in der Lebensqualitätsforschung. PPmP Psychother · Psychosom · Med Psychol. März 2002;52(3/4):141-50.

143. Tarlov AR, Ware JE, Greenfield S, Nelson EC, Perrin E, Zubkoff M. The Medical Outcomes Study. An application of methods for monitoring the results of medical care. JAMA. 18. August 1989;262(7):925-30.

144. Hays RD, Kallich JD, Mapes DL, Coons SJ, Carter WB. Development of the kidney disease quality of life (KDQOL) instrument. Qual Life Res Int J Qual Life Asp Treat Care Rehabil. Oktober 1994;3(5):329-38.

145. Janssen IM, Gerhardus A, Gersdorff GD von, Baldamus CA, Schaller M, Barth C, u. a. Preferences of patients undergoing hemodialysis \&ndash; results from a questionnairebased study with 4,518 patients [Internet]. Patient Preference and Adherence. 2015 [zitiert 24. Juni 2018]. Verfügbar unter: https://www.dovepress.com/preferences-of-patientsundergoing-hemodialysis-ndash-results-from-a-q-peer-reviewed-fulltext-article-PPA

146. Urquhart-Secord R, Craig JC, Hemmelgarn B, Tam-Tham H, Manns B, Howell M, u. a. Patient and Caregiver Priorities for Outcomes in Hemodialysis: An International Nominal Group Technique Study. Am J Kidney Dis. 1. September 2016;68(3):444-54.

147. Kutner NG, Zhang R, Huang Y, Johansen KL. Depressed mood, usual activity level, and continued employment after starting dialysis. Clin J Am Soc Nephrol CJASN. November 2010;5(11):2040-5.

148. Puñal Rioboó J, Sánchez-Iriso E, Ruano-Ravina A, Varela Lema ML, Sánchez-Guisande D, González-Rodríguez L, u. a. Short daily versus conventional hemodialysis quality of life: a cross-sectional multicentric study in Spain. Blood Purif. 2009;28(3):159-64.

149. Jaber BL, Lee Y, Collins AJ, Hull AR, Kraus MA, McCarthy J, u. a. Effect of daily hemodialysis on depressive symptoms and postdialysis recovery time: interim report from the FREEDOM (Following Rehabilitation, Economics and Everyday-Dialysis Outcome Measurements) Study. Am J Kidney Dis Off J Natl Kidney Found. September 2010;56(3):531-9.

150. Jansen MAM, Hart AAM, Korevaar JC, Dekker FW, Boeschoten EW, Krediet RT, u. a. Predictors of the rate of decline of residual renal function in incident dialysis patients. Kidney Int. September 2002;62(3):1046-53.

151. Achinger SG, Ikizler TA, Bian A, Shintani A, Ayus JC. Long-term effects of daily hemodialysis on vascular access outcomes: a prospective controlled study. Hemodial Int Int Symp Home Hemodial. April 2013;17(2):208-15.

152. Suri RS, Larive B, Hall Y, Kimmel PL, Kliger AS, Levin N, u. a. Effects of frequent hemodialysis on perceived caregiver burden in the Frequent Hemodialysis Network trials. Clin J Am Soc Nephrol CJASN. Mai 2014;9(5):936-42.

153. Suri RS, Li L, Nesrallah GE. The risk of hospitalization and modality failure with home dialysis. Kidney Int. August 2015;88(2):360-8.

154. Al Shakarchi J, Day C, Inston N. Vascular access for home haemodialysis. J Vasc Access. November 2018;19(6):593-5.

155. Miller BW, Himmele R, Sawin D-A, Kim J, Kossmann RJ. Choosing Home Hemodialysis: A Critical Review of Patient Outcomes. Blood Purif. 2018;45(1-3):224-9. 
156. Online-Version des EBM [Internet]. 2018 [zitiert 30. Juni 2018]. Verfügbar unter: http://www.kbv.de/html/online-ebm.php

157. Walker R, Marshall MR, Morton RL, McFarlane P, Howard K. The cost-effectiveness of contemporary home haemodialysis modalities compared with facility haemodialysis: a systematic review of full economic evaluations. Nephrol Carlton Vic. August 2014;19(8):45970 .

158. Mowatt G, Vale L, Perez J, Wyness L, Fraser C, MacLeod A, u. a. Systematic review of the effectiveness and cost-effectiveness, and economic evaluation, of home versus hospital or satellite unit haemodialysis for people with end-stage renal failure. Health Technol Assess Winch Engl. 2003;7(2):1-174.

159. Netzwerk Assistierte Dialyse [Internet]. [zitiert 3. Juli 2018]. Verfügbar unter: https://www.pflegewerk.com/unternehmen/kooperationspartner/pmc/netzwerk-assistiertedialyse.html

160. Bevilacqua MU, Turnbull L, Saunders S, Er L, Chiu H, Hill P, u. a. Evaluation of a 12Month Pilot of Long-Term and Temporary Assisted Peritoneal Dialysis. Perit Dial Int J Int Soc Perit Dial. Juni 2017;37(3):307-13.

161. Dratwa M. Costs of home assistance for peritoneal dialysis: results of a European survey. Kidney Int Suppl. April 2008;(108):72-75. 


\section{Erklärung}

$\S 4$ Abs. 3 (k) der HabOMed der Charité

Hiermit erkläre ich, dass

- weder früher noch gleichzeitig ein Habilitationsverfahren durchgeführt oder angemeldet wurde,

- die vorgelegte Habilitationsschrift ohne fremde Hilfe verfasst, die beschriebenen Ergebnisse selbst gewonnen sowie die verwendeten Hilfsmittel, die Zusammenarbeit mit anderen Wissenschaftlern/Wissenschaftlerinnen und mit technischen Hilfskräften sowie die verwendete Literatur vollständig in der Habilitationsschrift angegeben wurden,

- mir die geltende Habilitationsordnung bekannt ist. Ich erkläre ferner, dass mir die Satzung der Charité - Universitätsmedizin Berlin zur Sicherung Guter Wissenschaftlicher Praxis bekannt ist und ich mich zur Einhaltung dieser Satzung verpflichte.

22.1.2019

Datum Unterschrift 


\section{Danksagung}

Ich möchte mich als erstes ganz besonders herzlich bei Herrn Professor Dominik Müller bedanken. Er hat meine klinische und wissenschaftliche Ausbildung von Anfang an mit begleitet. Durch seine eigene Begeisterung für die medizinische Forschung und sein Ideenreichtum hat er mein wissenschaftliches Arbeiten geprägt. Seine fortwährende Unterstützung, sein stetes Interesse an meinen Ideen und sein kritisches, humorvolles Nachfragen haben diese Arbeit überhaupt nur möglich gemacht.

Das Projekt der Berliner Nachtdialyse für Kinder und Jugendliche wäre ohne den persönlichen nächtlichen Einsatz von Frau Dr. Zimmering, Herrn Professor Müller und den damaligen Pflegekräften der Kinderdialyse nicht möglich gewesen. Ihnen gilt mein besonderer Dank.

Als nächstes möchte ich Herrn Professor Bufler danken, der mich als Direktor der Klinik für Pädiatrie mit Schwerpunkten Gastroenterologie, Nephrologie und Stoffwechselmedizin in meinen Forschungsvorhaben voll unterstützt hat. Durch seine Aufgeschlossenheit, seine Verbindlichkeit und seine Wertschätzung allen Mitarbeitern gegenüber hat er in der vor zwei Jahren neu gegründeten Klinik eine sehr angenehme, produktive Arbeitsatmosphäre geschaffen.

Mein Dank gilt Herrn Professor Querfeld, der mich in den ersten Jahren meiner Klinik- und Forschungstätigkeit als Direktor der Klinik für Pädiatrie mit Schwerpunkt Nephrologie voll unterstützt hat. Durch zwischenzeitliche Freistellung von der klinischen Tätigkeit war es mir möglich, meine Forschungsprojekte zu beginnen.

Ganz besonders bedanken möchte ich mich auch bei Herrn Professor Pommer. Dank inm habe ich gelernt, dass es viele gemeinsame Fragestellungen im Bereich der Pädiatrie und Geriatrie gibt. Ohne seinen Beistand, seine Begeisterung und Aufmunterung wäre diese Arbeit nicht in der Form möglich gewesen.

Ich möchte mich bei allen jetzigen und ehemaligen Mitarbeiterinnen und Mitarbeitern der Abteilung bedanken, die meine Arbeit immer wohlwollend unterstützt haben und bereit waren, mir Freiräume für meine wissenschaftliche Arbeit zu gewähren. Mein Dank gilt allen Kooperationspartnern der einzelnen Projekte. Ohne gemeinsames Arbeiten und Diskutieren wären solche Vorhaben nicht zu realisieren.

Meiner Freundin, Frau Dr. Christine Schürmann, möchte ich herzlich danken für das Korrekturlesen dieser Arbeit.

Mein ganz besonderer inniger Dank gilt meinen Freunden und meiner Familie. An dieser Stelle möchte ich mich aus tiefen Herzen bei meinem Mann, Herrn Dr. Steffen Wagner, bedanken, der mich in allen Lebenslagen seit über 20 Jahren immer voll unterstützt, nicht zuletzt mit statistischem Wissen. Mit inm an der Seite ist es möglich, Beruf und Familie gemeinsam zu realisieren. 